\title{
CONVERGENCE RATES FOR AN INEXACT ADMM APPLIED TO SEPARABLE CONVEX OPTIMIZATION *
}

\author{
WILLIAM W. HAGER ${ }^{\dagger}$ AND HONGCHAO ZHANG Z $^{\ddagger}$
}

\begin{abstract}
Convergence rates are established for an inexact accelerated alternating direction method of multipliers (I-ADMM) for general separable convex optimization with a linear constraint. Both ergodic and non-ergodic iterates are analyzed. Relative to the iteration number $k$, the convergence rate is $\mathcal{O}(1 / k)$ in a convex setting and $\mathcal{O}\left(1 / k^{2}\right)$ in a strongly convex setting. When an error bound condition holds, the algorithm is 2-step linearly convergent. The I-ADMM is designed so that the accuracy of the inexact iteration preserves the global convergence rates of the exact iteration, leading to better numerical performance in the test problems.
\end{abstract}

Key words. Separable convex optimization; Alternating direction method of multipliers; ADMM; Accelerated gradient method; Inexact methods; Global convergence; Convergence rates

AMS subject classifications. 90C06, 90C25, 65Y20

1. Introduction. We consider a convex, separable linearly constrained optimization problem

$$
\min \Phi(\mathbf{x}) \text { subject to } \mathbf{A x}=\mathbf{b},
$$

where $\Phi: \mathbb{R}^{n} \rightarrow \mathbb{R} \cup\{\infty\}$ and $\mathbf{A}$ is $N$ by $n$. By a separable convex problem, we mean that the objective function is a sum of $m$ independent parts, and the matrix is partitioned compatibly as in

$$
\Phi(\mathbf{x})=\sum_{i=1}^{m} f_{i}\left(\mathbf{x}_{i}\right)+h_{i}\left(\mathbf{x}_{i}\right) \text { and } \mathbf{A} \mathbf{x}=\sum_{i=1}^{m} \mathbf{A}_{i} \mathbf{x}_{i} .
$$

Here $f_{i}$ is convex and Lipschitz continuously differentiable, $h_{i}$ is a proper closed convex function (possibly nonsmooth), and $\mathbf{A}_{i}$ is $N$ by $n_{i}$ with $\sum_{i=1}^{m} n_{i}=n$. There is no column independence assumption for the $\mathbf{A}_{i}$. Constraints of the form $\mathbf{x}_{i} \in \mathcal{X}_{i}$, where $\mathcal{X}_{i}$ is a closed convex set, can be incorporated in the optimization problem by letting $h_{i}$ be the indicator function of $\mathcal{X}_{i}$. That is, $h_{i}\left(\mathbf{x}_{i}\right)=\infty$ when $\mathbf{x}_{i} \notin \mathcal{X}_{i}$. The problem (1.1) - (1.2) has attracted extensive research due to its importance in areas such as image processing, statistical learning, and compressed sensing. See the recent survey 2 and its references.

It is assumed that there exists a solution $\mathrm{x}^{*}$ to (1.1)-1.2) and an associated Lagrange multiplier $\boldsymbol{\lambda}^{*} \in \mathbb{R}^{N}$ such that the following first-order optimality conditions hold: $\mathbf{A x}^{*}=\mathbf{b}$ and for $i=1,2, \ldots, m$ and for all $\mathbf{u} \in \mathbb{R}^{n_{i}}$, we have

$$
\left\langle\nabla f_{i}\left(\mathbf{x}_{i}^{*}\right)+\mathbf{A}_{i}^{\top} \boldsymbol{\lambda}^{*}, \mathbf{u}-\mathbf{x}_{i}^{*}\right\rangle+h_{i}(\mathbf{u}) \geq h_{i}\left(\mathbf{x}_{i}^{*}\right),
$$

where $\nabla$ denotes the gradient.

* January 12, 2020, revised June 1, 2020. The authors gratefully acknowledge support by the National Science Foundation under grants 1819002 and 1819161, and by the Office of Naval Research under grants N00014-15-1-2048 and N00014-18-1-2100.

†hager@ufl.edu, http://people.clas.ufl.edu/hager/, PO Box 118105, Department of Mathematics, University of Florida, Gainesville, FL 32611-8105. Phone (352) 294-2308. Fax (352) 392-8357.

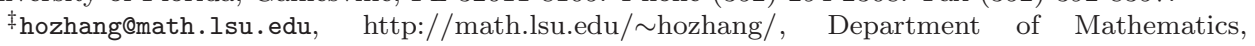
Louisiana State University, Baton Rouge, LA 70803-4918. Phone (225) 578-1982. Fax (225) 578-4276. 
A popular strategy for solving (1.1)-(1.2) is the alternating direction method of multipliers (ADMM) [16, 17]: For $i=1, \ldots, m$,

$$
\left\{\begin{array}{l}
\mathbf{x}_{i}^{k+1} \in \arg \min _{\mathbf{x}_{i} \in \mathbb{R}^{n_{i}}} \mathcal{L}_{\rho}\left(\mathbf{x}_{1}^{k+1}, \ldots, \mathbf{x}_{i-1}^{k+1}, \mathbf{x}_{i}, \mathbf{x}_{i+1}^{k}, \ldots, \mathbf{x}_{m}^{k}, \boldsymbol{\lambda}^{k}\right), \\
\boldsymbol{\lambda}^{k+1}=\boldsymbol{\lambda}^{k}+\rho\left(\mathbf{A} \mathbf{x}^{k+1}-\mathbf{b}\right),
\end{array}\right.
$$

where $\rho$ is a penalty parameter and $\mathcal{L}_{\rho}$ is the augmented Lagrangian defined by

$$
\mathcal{L}_{\rho}(\mathbf{x}, \boldsymbol{\lambda})=\Phi(\mathbf{x})+\langle\boldsymbol{\lambda}, \mathbf{A} \mathbf{x}-\mathbf{b}\rangle+\frac{\rho}{2}\|\mathbf{A} \mathbf{x}-\mathbf{b}\|^{2} .
$$

Early ADMMs only consider problem (1.1)-(1.2) with $m=2$ corresponding to a 2-block structure. In this case, the global convergence and complexity can be found in 12, 28. When $m \geq 3$, the ADMM strategy (1.4) is not necessarily convergent 4, although its practical efficiency has been observed in many recent applications [40, 41]. Many recent papers, including [3, 5, 6, 11, 18, 24, 26, 27, 32, 33, develop modifications to ADMM to ensure convergence when $m \geq 3$. The approach we have taken employs a back substitution step to complement the ADMM forward substitution step. This modification was first introduced in [26, 27.

Much of the CPU time in an ADMM iteration is associated with the solution of the minimization subproblems. If $m=1$, then ADMM reduces to the augmented Lagrangian method, for which the first relative error criteria based on the residual in an iteration emanates from [37, while more recent work includes [13, 39. For $m=2$ or larger, inexact approaches to the ADMM subproblems have been based on an absolute summable error criterion as in 9, 12, 19, a combined adaptive/absolute summable error criterion [31, a relative error criteria [14, 15], proximal regularizations [7. 25], and linearized subproblems and reduced multiplier update steps [30.

The approach taken in our I-ADMM emanates from our earlier work 10, 20, 21 on a Bregman Operator Splitting algorithm with a variable stepsize (BOSVS) with application to image processing. In the current paper, the penalty term in the accelerated gradient algorithm of 21] is linearized so as to make the solution of the I-ADMM subproblem trivial; there is essentially no reduction in the size of the multiplier update step. The I-ADMM is designed so that the accuracy of the inexact solution of the ADMM subproblems is high enough to preserve the global convergence rates of the exact iteration. The global convergence results for I-ADMM are similar to those presented in 21. However, there is no convergence rate analysis in 21. In this paper, we focus on the convergence rate of I-ADMM. In particular, relative to the iteration number $k$, the convergence rate for I-ADMM is $\mathcal{O}(1 / k)$ for ergodic iterates in the convex setting and $\mathcal{O}\left(1 / k^{2}\right)$ for both ergodic and nonergodic iterates in a strongly convex setting. When an error bound condition holds, I-ADMM is 2-step linearly convergent. These convergence rates are consistent with those obtained for ADMM schemes that solve subproblems exactly including the $\mathcal{O}(1 / k)$ rates in [28, 35, 38] for ergodic iterates, and the linear rates obtained in 23 and 42 for a 2-block ADMM, and in [30] for the multi-block case and a sufficiently small stepsize in the multiplier update. For a more extensive review of linear convergence results for ADMMs, see [43. But again, almost all the sublinear or linear convergence rate analysis is based on either a single linearization step to solve the subproblem or the exact solution of the (proximal) subproblem. An advantage of our inexact scheme, compared to the exact iteration, is that the computing time to achieve a given error tolerance is reduced, while maintaining global convergence and its rate. 
The paper is organized as follows. Section 2 gives an overview of the inexact ADMM (I-ADMM) that will be analyzed. Section 3 reviews the global convergence results found in a companion paper 22]. These global convergence results are similar to those established for the inexact ADMM of [21. Section 4 establishes a $\mathcal{O}(1 / k)$ convergence rate of for ergodic iterates, and under a strong convexity assumption, an $\mathcal{O}\left(1 / k^{2}\right)$ rate for both ergodic and nonergodic iterates. Section 5 gives 2-step linear convergence results when an error bound condition holds. Finally, Section 6 shows the observed convergence in some image recovery problems.

1.1. Notation. Throughout the paper, $c$ denotes a generic positive constant which is independent of parameters such as the iteration number $k$ or the index $i \in[1, m]$. Let $\mathcal{W}^{*}$ denote the set of solution/multiplier pairs $\left(\mathbf{x}^{*}, \boldsymbol{\lambda}^{*}\right)$ of (1.1)-(1.2) satisfying (1.3), while $\left(\mathrm{x}^{*}, \boldsymbol{\lambda}^{*}\right) \in \mathcal{W}^{*}$ is a generic solution/multiplier pair. $\mathcal{L}$ (without the $\rho$ subscript) stands for $\mathcal{L}_{0}$. For $\mathbf{x}$ and $\mathbf{y} \in \mathbb{R}^{n},\langle\mathbf{x}, \mathbf{y}\rangle=\mathbf{x}^{\top} \mathbf{y}$ is the standard inner product, where the superscript ${ }^{\top}$ denotes transpose. The Euclidean vector norm, denoted $\|\cdot\|$, is defined by $\|\mathbf{x}\|=\sqrt{\langle\mathbf{x}, \mathbf{x}\rangle}$ and $\|\mathbf{x}\|_{\mathbf{G}}=\sqrt{\mathbf{x}^{\top} \mathbf{G} \mathbf{x}}$ for a positive definite matrix $\mathbf{G}$. For any matrix $\mathbf{A}$, the matrix norm induced by the Euclidean vector norm is the largest singular value of $\mathbf{A}$. For a symmetric matrix, the Euclidean norm is the largest absolute eigenvalue. In addition, $\mathbf{A} \succ \mathbf{0}$ and $\mathbf{A} \succeq \mathbf{0}$ mean that the matrix $\mathbf{A}$ is positive definite and positive semidefinite, respectively. For a differentiable function $f: \mathbb{R}^{n} \rightarrow \mathbb{R}, \nabla f(\mathbf{x})$ is the gradient of $f$ at $\mathbf{x}$, a column vector. More generally, $\partial f(\mathbf{x})$ denotes the subdifferential at $\mathbf{x}$. A function $h: \mathbb{R}^{n} \mapsto \mathbb{R}$ is convex with modulus $\mu \geq 0$ if

$$
h((1-\theta) \mathbf{x}+\theta \mathbf{y}) \leq(1-\theta) h(\mathbf{x})+\theta h(\mathbf{y})-\theta(1-\theta)(\mu / 2)\|\mathbf{x}-\mathbf{y}\|^{2}
$$

for all $\mathbf{u}$ and $\mathbf{v} \in \mathbb{R}^{n}$ and $\theta \in[0,1]$. If $\mu>0$, then $h$ is strongly convex. The prox operator associated with $h$ is defined by

$$
\operatorname{prox}_{h}(\mathbf{y})=\arg \min _{\mathbf{x} \in \mathbb{R}^{n}}\left(h(\mathbf{x})+\frac{1}{2}\|\mathbf{x}-\mathbf{y}\|^{2}\right) .
$$

2. Algorithm Structure. The structure of our I-ADMM algorithm is given in Algorithm 2.1. The algorithm generates sequences $\mathbf{x}^{k}, \mathbf{y}^{k}, \mathbf{z}^{k}$, and $R^{k}$. Both $\mathbf{x}^{k}$ and $\mathbf{z}^{k}$ are updated in Step 1, $R^{k}$ is updated in Step 2, and $\mathbf{y}^{k}$ is updated in Step 3. The error is estimated in Step 2. The matrix $\mathbf{Q}$ in Step 3 is an $m$ by $m$ block diagonal matrix whose $i$-th diagonal block, denoted $\mathbf{Q}_{i}$, is chosen to satisfy the conditions:

$$
\mathbf{Q}_{i} \succ \mathbf{0} \quad \text { and } \quad \overline{\mathbf{Q}}_{i}:=\mathbf{Q}_{i}-\mathbf{A}_{i}^{\top} \mathbf{A}_{i} \succeq \mathbf{0} .
$$

For example, we could take $\mathbf{Q}_{i}=\gamma_{i} \mathbf{I}$ where $\gamma_{i} \geq\left\|\mathbf{A}_{i}^{\top} \mathbf{A}_{i}\right\|$. Condition (2.1) is required for showing global convergence of our I-ADMM. Recent studies show that for the 2-block case $(m=2)$ and an exact ADMM, the requirement that $\overline{\mathbf{Q}}_{i}$ is positive semidefinite can be relaxed [ 8,29 . The matrix $\mathbf{M}$ in Step 3 is the $m$ by $m$ block lower triangular matrix defined by

$$
\mathbf{M}_{i j}=\left\{\begin{array}{cc}
\mathbf{A}_{i}^{\top} \mathbf{A}_{j} & \text { if } j<i, \\
\mathbf{Q}_{i} & \text { if } j=i, \\
\mathbf{0} & \text { if } j>i .
\end{array}\right.
$$

By (2.1), $M$ is nonsingular. The solution $\mathbf{y}^{k+1}$ of the block upper triangular system $\mathbf{M}^{\top}\left(\mathbf{y}^{k+1}-\mathbf{y}^{k}\right)=\alpha \mathbf{Q}\left(\mathbf{z}^{k}-\mathbf{y}^{k}\right)$ can be obtained by back substitution. 


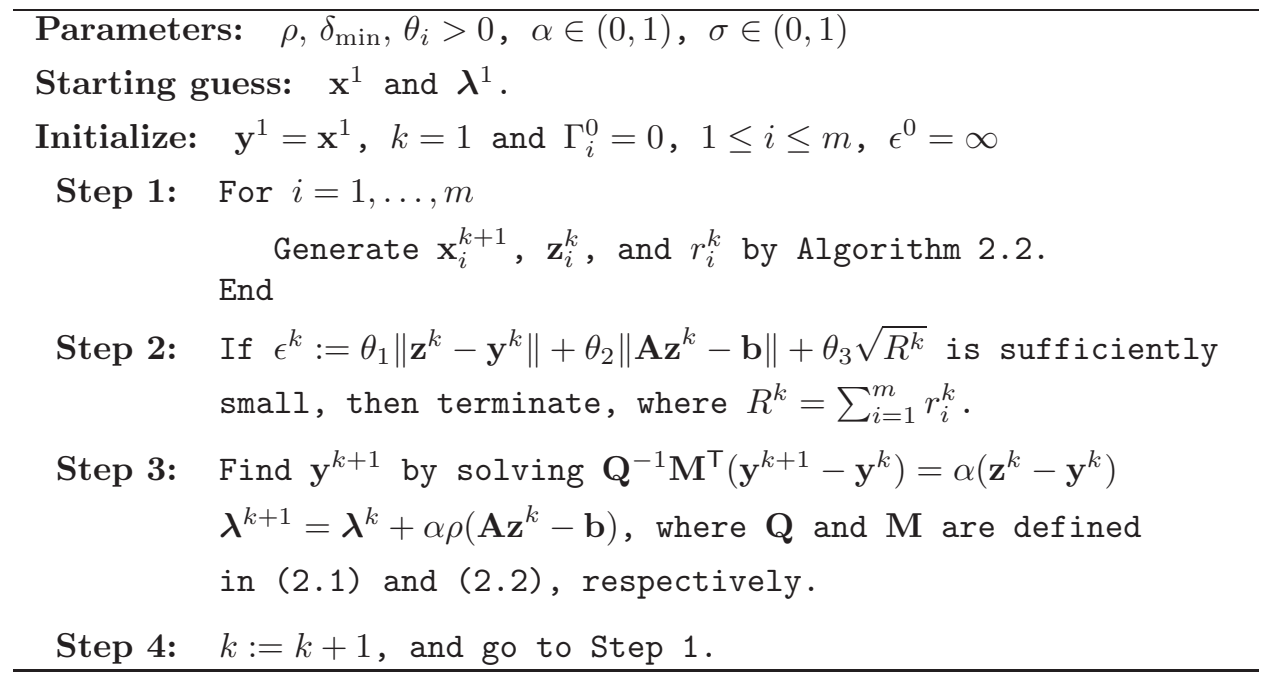

ALG. 2.1. I-ADMM algorithm.

In Step 1 of Algorithm 2.1, we approximate the minimizer in the $\mathbf{x}_{i}$ subproblem of the ADMM algorithm (1.4) using the accelerated gradient method of Algorithm 2.2 which is a modification of Algorithm 5.1 in [22]. Compared with Algorithm 5.1 in [22], Algorithm 2.2 has a slightly different stopping condition in Step 1b, and a proximal term to generate $\mathbf{u}_{i}^{l}$ in Step 1a, where

$$
\mathbf{b}_{i}^{k}=\mathbf{b}-\sum_{j<i} \mathbf{A}_{j} \mathbf{z}_{j}^{k}-\sum_{j>i} \mathbf{A}_{j} \mathbf{y}_{j}^{k} .
$$

The termination condition for Algorithm 2.2 appears in Step 1b. In this step, $\psi$ is a nonnegative function for which $\psi(0)=0$ and $\psi(s)>0$ for $s>0$ with $\psi$ continuous at $s=0$. For example, $\psi(t)=t$. Two different ways are developed in 21] for choosing the parameters $\delta^{l}$ and $\alpha^{l}$ in Step 1a. If a Lipschitz constant $\zeta_{i}$ of $f_{i}$ is known, then we could take

$$
\delta^{l}=\frac{1}{(1-\sigma)} \frac{2 \zeta_{i}}{l} \quad \text { and } \quad \alpha^{l}=\frac{2}{l+1} \in(0,1],
$$

in which case, we have

$$
\frac{(1-\sigma) \delta^{l}}{\alpha^{l}}=\frac{(l+1) \zeta_{i}}{l}>\zeta_{i} .
$$

This relation along with a Taylor series expansion of $f_{i}$ around $\overline{\mathbf{a}}_{i}^{l}$ implies that the line search condition in Step 1a of Algorithm 2.2 is satisfied for each $l$.

A different, adaptive way to choose to choose $\delta^{l}$ and $\alpha^{l}$, that does not require knowledge of the Lipschitz constant for $f_{i}$, is the following: Choose $\delta_{0}^{l} \in\left[\delta_{\min }, \delta_{\max }\right]$, where $0<\delta_{\min }<\delta_{\max }<\infty$ are fixed constants, independent of $k$ and $l$, and set

$$
\begin{aligned}
\delta^{l} & =\frac{2}{\theta^{l}+\sqrt{\left(\theta^{l}\right)^{2}+4 \theta^{l} \Lambda^{l-1}}} \quad \text { and } \quad \alpha^{l}=\frac{1}{1+\delta^{l} \Lambda^{l-1}}, \quad \text { where } \\
\Lambda^{l} & =\sum_{i=1}^{l} 1 / \delta^{i}, \quad \Lambda^{0}=0, \quad \text { and } \quad \theta^{l}=1 /\left(\delta_{0}^{l} \eta^{j}\right) \text { with } \eta>1 .
\end{aligned}
$$




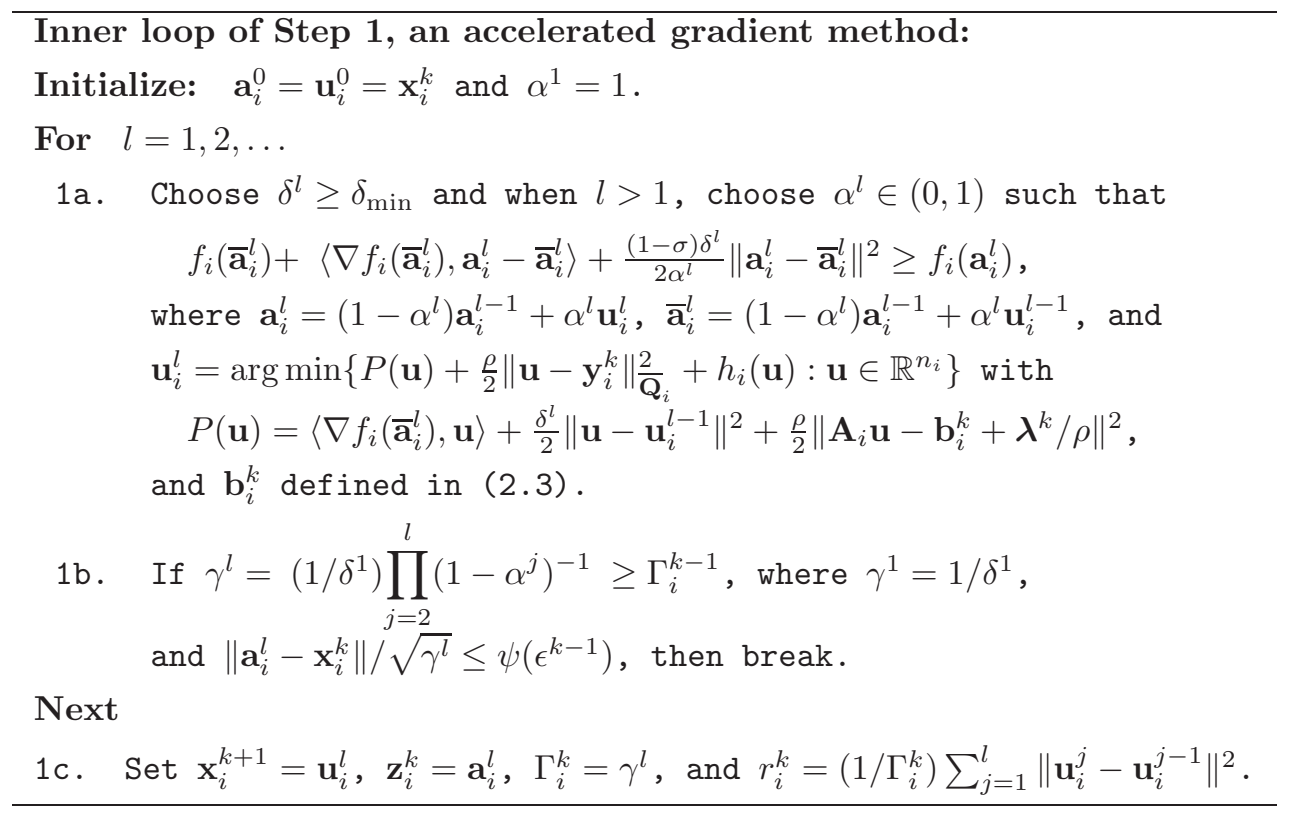

Alg. 2.2. Inner loop in Step 1 of Algorithm 2.1.

Here the integer $j \geq 0$ is chosen as small a possible while satisfying the inequality in Step 1a. It can be shown that

$$
\frac{\delta^{l}}{\alpha^{l}}=\frac{1}{\theta^{l}}=\delta_{0}^{l} \eta^{j}
$$

Since $\eta>1$, the ratio $\delta^{l} / \alpha^{l}$ appearing in Step 1a tends to infinity as $j$ tends to infinity; consequently, the inequality in Step 1a is satisfied for $j$ sufficiently large.

The stopping condition in Step 1b is elucidated using the following function:

$$
\begin{aligned}
& \bar{L}_{i}^{k}(\mathbf{u})=L_{i}^{k}(\mathbf{u})+\frac{\rho}{2}\left(\mathbf{u}-\mathbf{y}_{i}^{k}\right)^{\top} \overline{\mathbf{Q}}_{i}\left(\mathbf{u}-\mathbf{y}_{i}^{k}\right), \quad \text { where } \\
& L_{i}^{k}(\mathbf{u})=f_{i}(\mathbf{u})+h_{i}(\mathbf{u})+\frac{\rho}{2}\left\|\mathbf{A}_{i} \mathbf{u}-\mathbf{b}_{i}^{k}+\boldsymbol{\lambda}^{k} / \rho\right\|^{2},
\end{aligned}
$$

and $\mathbf{b}_{i}^{k}$ is defined in (2.3). As pointed out in Lemma 3.1 of the next section, for either of the parameter choices (2.4) or (2.5), the iterates $\mathbf{a}_{i}^{l}$ of Algorithm 2.2 converge to the minimizer of the function $\bar{L}_{i}^{k}$ at rate $\mathcal{O}(1 / l)$, while the objective values converge at rate $\mathcal{O}\left(1 / l^{2}\right)$, which is optimal for first-order methods applied to general convex, possibly nonsmooth optimization problems. We let $l_{i}^{k}$ denote the terminating value of $l$ in Step 1b.

REMARK 2.1. For the two parameter choices (2.4) and (2.5), it has been shown [21, pp. 227-228] that in Step $1 \mathrm{~b}, \gamma^{l} \geq l^{2} \Theta$ for some constant $\Theta>0$, independent of $k$ and $l$. Consequently, the conditions in Step $1 \mathrm{~b}$ are satisfied for $l$ sufficiently large.

3. Global Convergence. The global convergence analysis of the accelerated ADMM in this paper with a linearized penalty term is similar to the global convergence analysis of the accelerated scheme in 21. Hence, this section simply states the main results, while the Appendix provides the detailed analysis. The first result concerns 
the convergence of the iterates in Step 1 of I-ADMM under the assumption that the sequence

$$
\xi^{l}:=\delta^{l} \alpha^{l} \gamma^{l}
$$

is nondecreasing. For either of the parameter choices (2.4) or (2.5), it is shown in 21, pp. 227-228] that $\xi^{l}=1$.

LEMMA 3.1. If the sequence $\xi^{l}$ is nonincreasing, then for each $i \in[1, m]$ and $L \geq 1$, we have

$$
\rho \nu_{i}\left\|\mathbf{a}_{i}^{L}-\overline{\mathbf{x}}_{i}^{k}\right\|^{2}+\frac{\mu_{h, i}}{2} \sum_{l=1}^{L}\left\|\overline{\mathbf{x}}_{i}^{k}-\mathbf{a}_{i}^{L}\right\|^{2}+\frac{\sigma}{\gamma^{L}} \sum_{l=1}^{L} \xi^{l}\left\|\mathbf{u}_{i}^{l}-\mathbf{u}_{i}^{l-1}\right\|^{2} \leq \frac{\left\|\mathbf{x}_{i}^{k}-\overline{\mathbf{x}}_{i}^{k}\right\|^{2}}{\gamma^{L}}
$$

where $\mu_{h, i}$ is the modulus of convexity of $h_{i}, \nu_{i}>0$ is the smallest eigenvalue of $\mathbf{Q}_{i}$, and

$$
\overline{\mathbf{x}}_{i}^{k}=\arg \min \left\{\bar{L}_{i}^{k}(\mathbf{u}): \mathbf{u} \in \mathbb{R}^{n_{i}}\right\}
$$

Since $\bar{L}_{i}^{k}$ is strongly convex, it has a unique minimizer. The following decay property plays an important role in the global convergence analysis.

LEMMA 3.2. Let $\left(\mathbf{x}^{*}, \boldsymbol{\lambda}^{*}\right) \in \mathcal{W}^{*}$ be any solution/multiplier pair for (1.1)-(1.2), let $\mathbf{x}^{k}, \mathbf{y}^{k}, \mathbf{z}^{k}, \mathbf{u}_{k}^{l}$, and $\boldsymbol{\lambda}^{k}$ be the iterates generated by Algorithm 2.1 , and define

$$
\begin{aligned}
& E_{k}=\rho\left\|\mathbf{y}^{k}-\mathbf{x}^{*}\right\|_{\mathbf{P}}^{2}+\frac{1}{\rho}\left\|\boldsymbol{\lambda}^{k}-\boldsymbol{\lambda}^{*}\right\|^{2}+\alpha \sum_{i=1}^{m} \frac{\left\|\mathbf{x}_{i}^{k}-\mathbf{x}_{i}^{*}\right\|^{2}}{\Gamma_{i}^{k}} \text { and } \\
& E_{k}^{-}=\rho\left\|\mathbf{y}^{k}-\mathbf{x}^{*}\right\|_{\mathbf{P}}^{2}+\frac{1}{\rho}\left\|\boldsymbol{\lambda}^{k}-\boldsymbol{\lambda}^{*}\right\|^{2}+\alpha \sum_{i=1}^{m} \frac{\left\|\mathbf{x}_{i}^{k}-\mathbf{x}_{i}^{*}\right\|^{2}}{\Gamma_{i}^{k-1}}
\end{aligned}
$$

where $\mathbf{P}=\mathbf{M Q}^{-1} \mathbf{M}^{\top}$. If $\xi^{l}:=\delta^{l} \alpha^{l} \gamma^{l}=1$ for each $l$, then

$$
\begin{gathered}
E_{k}-E_{k+1} \geq E_{k}-E_{k+1}^{-} \geq \\
\alpha\left(2 \Delta^{k}+\sigma R^{k}+\rho(1-\alpha)\left(\left\|\mathbf{y}^{k}-\mathbf{z}^{k}\right\|_{\mathbf{Q}}^{2}+\left\|\mathbf{A} \mathbf{z}^{k}-\mathbf{b}\right\|^{2}\right)+\sum_{i=1}^{m} \mu_{h, i}\left\|\mathbf{z}_{i}^{k}-\mathbf{x}_{i}^{*}\right\|^{2}\right),
\end{gathered}
$$

where $R^{k}$ is the residual defined in Step 2, $\mu_{h, i}$ is the modulus of convexity of $h_{i}$, and

$$
\Delta^{k}=\mathcal{L}\left(\mathbf{z}^{k}, \boldsymbol{\lambda}^{*}\right)-\Phi\left(\mathbf{x}^{*}\right) \geq 0 .
$$

Recall that $\mathcal{L}=\mathcal{L}_{0}$ is the ordinary Lagrangian associated with (1.1). This decay property is used to obtain the following global convergence result for I-ADMM.

TheOREM 3.3. Suppose the parameters $\delta^{l}$ and $\alpha^{l}$ in Algorithm 2.2 are chosen according to either (2.4) or (2.5). If I-ADMM performs an infinite number of iterations generating $\mathbf{y}^{k}, \mathbf{z}^{k}$, and $\boldsymbol{\lambda}^{k}$, then the sequences $\mathbf{y}^{k}$ and $\mathbf{z}^{k}$ both approach a common limit $\mathbf{x}^{*}, \lambda^{k}$ approaches a limit $\boldsymbol{\lambda}^{*}$, and $\left(\mathbf{x}^{*}, \boldsymbol{\lambda}^{*}\right) \in \mathcal{W}^{*}$.

Theorem 3.3 considers the case of an infinite number of iterations. The following lemma considers the case where $\epsilon^{k}=0$ within a finite number of iterations. 
Lemma 3.4. If $\epsilon^{k}=0$ in Algorithm 2.1, then $\mathbf{x}^{k+1}=\mathbf{x}^{k}=\mathbf{y}^{k}=\mathbf{z}^{k}$ solves (1.1) -(1.2) and $\left(\mathrm{x}^{k}, \boldsymbol{\lambda}^{k}\right) \in \mathcal{W}^{*}$.

Proof. If $\epsilon^{k}=0$, then $r_{i}^{k}=0$ for each $i$. It follows that

$$
\mathbf{x}_{i}^{k}=\mathbf{u}_{i}^{0}=\mathbf{u}_{i}^{1}=\ldots=\mathbf{u}_{i}^{l} .
$$

By Step 1c, $\mathbf{u}_{i}^{l}=\mathbf{x}_{i}^{k+1}$. By the definitions $\mathbf{a}_{i}^{l}=\left(1-\alpha^{l}\right) \mathbf{a}_{i}^{l-1}+\alpha^{l} \mathbf{u}_{i}^{l}$ and $\overline{\mathbf{a}}_{i}^{l}=$ $\left(1-\alpha^{l}\right) \mathbf{a}_{i}^{l-1}+\alpha^{l} \mathbf{u}_{i}^{l-1}$ where $\mathbf{a}_{i}^{0}=\mathbf{u}_{i}^{0}=\mathbf{x}_{i}^{k}$, we have $\mathbf{a}_{i}^{l}=\overline{\mathbf{a}}_{i}^{l}=\mathbf{x}_{i}^{k}$ for each $l$ due to (3.6). Again, by Step $1 \mathrm{c}, \mathbf{z}_{i}^{k}=\mathbf{x}_{i}^{k}$. Consequently, we have $\mathbf{x}^{k+1}=\mathbf{x}^{k}=\mathbf{z}^{k}$.

Let $\mathbf{x}^{*}$ denote $\mathbf{x}^{k}$. Then $\mathbf{x}^{*}=\mathbf{x}^{k+1}=\mathbf{x}^{k}=\mathbf{z}^{k}$. Since $\epsilon^{k}=0$, Step 2 of Algorithm 2.1 implies that $\mathbf{y}^{k}=\mathbf{z}^{k}=\mathbf{x}^{*}$ and $\mathbf{A} \mathbf{x}^{*}=\mathbf{b}$. Consequently, we have

$$
\mathbf{b}_{i}^{k}=\mathbf{b}-\sum_{j<i} \mathbf{A}_{j} \mathbf{z}_{j}^{k}-\sum_{j>i} \mathbf{A}_{j} \mathbf{y}_{j}^{k}=\mathbf{b}-\sum_{j<i} \mathbf{A}_{j} \mathbf{x}_{j}^{*}-\sum_{j>i} \mathbf{A}_{j} \mathbf{x}_{j}^{*}=\mathbf{A}_{i} \mathbf{x}_{i}^{*} .
$$

With this substitution in $P(\mathbf{u})$ in Step 1a, it follows that $\mathbf{u}_{i}^{l}=\mathbf{x}_{i}^{*}$ minimizes over $\mathbf{u}$ the function

$$
\left\langle\nabla f_{i}\left(\mathbf{x}_{i}^{*}\right), \mathbf{u}\right\rangle+\frac{\delta^{l}}{2}\left\|\mathbf{u}-\mathbf{x}_{i}^{*}\right\|^{2}+\frac{\rho}{2}\left\|\mathbf{A}_{i}\left(\mathbf{u}-\mathbf{x}_{i}^{*}\right)+\lambda^{k} / \rho\right\|^{2}+\frac{\rho}{2}\left\|\mathbf{u}-\mathbf{x}_{i}^{*}\right\|_{\overline{\mathbf{Q}}_{i}}^{2}+h_{i}(\mathbf{u}) .
$$

The first-order optimality condition for this minimizer $\mathbf{x}_{i}^{*}$ is the same as the first-order optimality condition (1.3), but with $\boldsymbol{\lambda}^{*}$ replaced by $\boldsymbol{\lambda}^{k}$. Hence, $\left(\mathbf{x}^{*}, \boldsymbol{\lambda}^{k}\right) \in \mathcal{W}^{*}$.

REMARK 3.1. In this paper, we have focused on algorithms based on an inexact minimization of $\bar{L}_{i}^{k}$ in Step 1 of Algorithm 2.1. In cases where $f_{i}$ and $h_{i}$ are simple enough that the exact minimizer $\overline{\mathbf{x}}_{i}^{k}$ of $\bar{L}_{i}^{k}$ can be quickly evaluated, we could simply set $\mathbf{x}_{i}^{k+1}=\mathbf{z}_{i}^{k}=\overline{\mathbf{x}}_{i}^{k}$, and $r_{i}^{k}=0$ in Step 1 of I-ADMM, and proceed to Step 2. The global convergence results still hold.

4. Sublinear Convergence Rates. In this section, sublinear convergences rates are established for I-ADMM. We first establish an $\mathcal{O}(1 / t)$ convergence rate for the ergodic iterates

$$
\overline{\mathbf{z}}^{t}=\frac{1}{t} \sum_{k=1}^{t} \mathbf{z}^{k}
$$

generated by I-ADMM.

THEOREM 4.1. Let $\left(\mathbf{x}^{*}, \boldsymbol{\lambda}^{*}\right) \in \mathcal{W}^{*}$ be any primal/dual solution pair for (1.1)(1.2) and let $\mathbf{z}^{k}$ be generated by I-ADMM with $\delta^{l} \alpha^{l} \gamma^{l}=1$ for each $l$ and $k$. Then, we have

$$
\mathcal{L}\left(\overline{\mathbf{z}}^{t}, \boldsymbol{\lambda}^{*}\right)-\Phi\left(\mathbf{x}^{*}\right) \leq \frac{E_{1}}{2 \alpha t},
$$

where $\overline{\mathbf{z}}^{t}$ is defined in (4.1) and $E_{k}$ is defined in (3.3).

Proof. Discarding several nonnegative terms from (3.4), we have

$$
2 \alpha \Delta^{k}+E_{k+1} \leq E_{k} .
$$

Adding this inequality over $k$ between 1 and $t$ yields

$$
2 \alpha \sum_{k=1}^{t} \Delta^{k}+E_{t+1} \leq E_{1} .
$$


Hence, by the definition of $\Delta^{k}$ in (3.5), we have

$$
2 \alpha \sum_{k=1}^{t}\left[\mathcal{L}\left(\mathbf{z}^{k}, \boldsymbol{\lambda}^{*}\right)-\Phi\left(\mathbf{x}^{*}\right)\right] \leq E_{1}
$$

By the convexity of $\Phi$ and the definition (4.1), it follows that

$$
2 \alpha t\left[\mathcal{L}\left(\overline{\mathbf{z}}^{t}, \boldsymbol{\lambda}^{*}\right)-\Phi\left(\mathbf{x}^{*}\right)\right] \leq E_{1}
$$

This completes the proof.

Note that the minimum of $\mathcal{L}\left(\mathbf{x}, \boldsymbol{\lambda}^{*}\right)$ over $\mathbf{x} \in \mathbb{R}^{n}$ is attained at $\mathbf{x}=\mathbf{x}^{*}$, and $\mathcal{L}\left(\mathbf{x}^{*}, \boldsymbol{\lambda}^{*}\right)=\Phi\left(\mathbf{x}^{*}\right)$. Hence, Theorem 4.1 bounds the difference between $\mathcal{L}\left(\overline{\mathbf{z}}^{t}, \boldsymbol{\lambda}^{*}\right)$ and the minimum of $\mathcal{L}\left(\cdot, \boldsymbol{\lambda}^{*}\right)$. We will strengthen the convergence rate to $\mathcal{O}\left(1 / t^{2}\right)$ when a strong convexity assumption holds, and also obtain a convergence rate for nonergodic iterates.

Assumption 4.1. If $\mu_{f, i} \geq 0$ and $\mu_{h, i} \geq 0$ are the convexity moduli of $f_{i}$ and $h_{i}$ respectively, then

$$
\mu=\min \left\{\mu_{f, i}+3 \mu_{h, i}: i=1, \ldots, m\right\}>0 .
$$

In the following theorem, we suppose that at the $k$-th iteration, the penalty parameter $\rho$ is chosen in the following way:

$$
\rho_{k}=\left(k_{0}+k\right) \theta
$$

where

$$
\theta=\frac{\alpha \mu}{8\|\mathbf{P}\|} \quad \text { and } \quad k_{0}=\frac{4\left\|\mathbf{Q}^{-1 / 2} \mathbf{P} \mathbf{Q}^{-1 / 2}\right\|}{\alpha(1-\alpha)},
$$

with $\mu$ defined in Assumption 4.1 $\alpha \in(0,1)$ is the parameter in Algorithm 2.1 and $\mathbf{P}=\mathrm{MQ}^{-1} \mathbf{M}^{\top}$. We have the following theorem:

TheOREm 4.2. Let $\left(\mathrm{x}^{*}, \boldsymbol{\lambda}^{*}\right) \in \mathcal{W}^{*}$ be any solution/multiplier pair for (1.1) - (1.2), let $\mathbf{x}^{k}, \mathbf{y}^{k}, \mathbf{z}^{k}$ and $\boldsymbol{\lambda}^{k}$ be generated by I-ADMM, and assume that Assumption 4.1 holds and $\delta^{l} \alpha^{l} \gamma^{l}=1$ for each $l$ and $k$. Suppose that for every $k, \rho_{k}$ is given by (4.3) and $\Gamma_{i}^{k}$ satisfies

$$
\frac{k}{\Gamma_{i}^{k}} \geq \frac{k+1}{\Gamma_{i}^{k+1}}, \quad 1 \leq i \leq m .
$$

Then, for all $t>0$, we have

$$
\mathcal{L}\left(\tilde{\mathbf{z}}^{t}, \boldsymbol{\lambda}^{*}\right)-\Phi\left(\mathbf{x}^{*}\right) \leq \frac{2 \bar{c}}{\alpha\left[t(t+1)+2 k_{0} t\right]}
$$

and

$$
\left\|\mathbf{y}^{t+1}-\mathbf{x}^{*}\right\|^{2} \leq \frac{\bar{c}}{\left(t+k_{0}\right)^{2} \theta}
$$

where

$$
\tilde{\mathbf{z}}^{t}=\frac{2}{t(t+1)+2 k_{0} t} \sum_{k=1}^{t}\left(\left(k_{0}+k\right) \mathbf{z}^{k}\right),
$$


and

$$
\bar{c}=\frac{1}{\theta}\left\|\boldsymbol{\lambda}^{1}-\boldsymbol{\lambda}^{*}\right\|^{2}+\alpha\left(k_{0}+1\right) \sum_{i=1}^{m} \frac{\left\|\mathbf{x}_{i}^{1}-\mathbf{x}_{i}^{*}\right\|^{2}}{\Gamma_{i}^{1}}+k_{0}^{2} \theta\left\|\mathbf{y}^{1}-\mathbf{x}^{*}\right\|_{\mathbf{P}}^{2} .
$$

Proof. By Assumption 4.1 and the definition (3.5) of $\Delta^{k}$, we have

$$
\Delta^{k}=\mathcal{L}\left(\mathbf{z}^{k}, \boldsymbol{\lambda}^{*}\right)-\mathcal{L}\left(\mathbf{x}^{*}, \boldsymbol{\lambda}^{*}\right) \geq \sum_{i=1}^{m} \frac{\mu_{f, i}+\mu_{h, i}}{2}\left\|\mathbf{z}_{i}^{k}-\mathbf{x}_{i}^{*}\right\|^{2}=\sum_{i=1}^{m} \frac{\mu_{f, i}+\mu_{h, i}}{2}\left\|\mathbf{z}_{e, i}^{k}\right\|^{2},
$$

where $\mathbf{z}_{e}^{k}=\mathbf{z}^{k}-\mathbf{x}^{*}$. The inequality (3.4) of Lemma 3.2 relates the error in two consecutive iterations, where the $\rho$ in (3.4) is the penalty at iteration $k$. Combining this with the definition of $\mu$ in Assumption 4.1 we have

$$
\begin{aligned}
& \alpha\left(\Delta^{k}+\frac{\mu}{2}\left\|\mathbf{z}_{e}^{k}\right\|^{2}+\rho_{k}(1-\alpha)\left\|\mathbf{y}^{k}-\mathbf{z}^{k}\right\|_{\mathbf{Q}}^{2}\right) \\
\leq & \rho_{k}\left(\left\|\mathbf{y}_{e}^{k}\right\|_{\mathbf{P}}^{2}-\left\|\mathbf{y}_{e}^{k+1}\right\|_{\mathbf{P}}^{2}\right)+\frac{1}{\rho_{k}}\left(\left\|\boldsymbol{\lambda}_{e}^{k}\right\|^{2}-\left\|\boldsymbol{\lambda}_{e}^{k+1}\right\|^{2}\right)+\alpha \sum_{i=1}^{m} \frac{\left\|\mathbf{x}_{e, i}^{k}\right\|^{2}-\left\|\mathbf{x}_{e, i}^{k+1}\right\|^{2}}{\Gamma_{i}^{k}},
\end{aligned}
$$

where $\mathbf{x}_{e}^{k}=\mathbf{x}^{k}-\mathbf{x}^{*}, \mathbf{y}_{e}^{k}=\mathbf{y}^{k}-\mathbf{x}^{*}$, and $\boldsymbol{\lambda}_{e}^{k}=\boldsymbol{\lambda}^{k}-\boldsymbol{\lambda}^{*}$.

For any matrix $\mathbf{P}$, it follows from an eigendecomposition that

$$
\mathbf{x}^{\top} \mathbf{x} \geq \frac{\mathbf{x}^{\top} \mathbf{P} \mathbf{x}}{\|\mathbf{P}\|} \quad \text { and } \quad \mathbf{x}^{\top} \mathbf{Q} \mathbf{x} \geq \frac{\mathbf{x}^{\top} \mathbf{P} \mathbf{x}}{\left\|\mathbf{Q}^{-1 / 2} \mathbf{P} \mathbf{Q}^{-1 / 2}\right\|} .
$$

The second inequality is deduced from the first when $\mathbf{x}$ is replaced by $\mathbf{Q}^{1 / 2} \mathbf{x}$ and $\mathbf{P}$ is replaced by $\mathbf{Q}^{-1 / 2} \mathbf{P Q}^{-1 / 2}$. This yields the following lower bound for terms on the left side of (4.10):

$$
\begin{aligned}
\frac{\mu}{2}\left\|\mathbf{z}_{e}^{k}\right\|^{2}+\rho_{k}(1-\alpha)\left\|\mathbf{y}^{k}-\mathbf{z}^{k}\right\|_{\mathbf{Q}}^{2} & \geq \frac{\mu}{2\|\mathbf{P}\|}\left\|\mathbf{z}_{e}^{k}\right\|_{\mathbf{P}}^{2}+\frac{\rho_{k}(1-\alpha)}{\left\|\mathbf{Q}^{-1 / 2} \mathbf{P} \mathbf{Q}^{-1 / 2}\right\|}\left\|\mathbf{y}^{k}-\mathbf{z}^{k}\right\|_{\mathbf{P}}^{2} \\
& \geq \frac{\mu}{2\|\mathbf{P}\|}\left(\left\|\mathbf{z}_{e}^{k}\right\|_{\mathbf{P}}^{2}+\left\|\mathbf{y}^{k}-\mathbf{z}^{k}\right\|_{\mathbf{P}}^{2}\right) \\
& \geq \frac{\mu}{2\|\mathbf{P}\|}\left(2\left\|\mathbf{z}_{e}^{k}\right\|_{\mathbf{P}}^{2}+\left\|\mathbf{y}_{e}^{k}\right\|_{\mathbf{P}}-2\left\|\mathbf{z}_{e}^{k}\right\|\left\|\mathbf{y}_{e}^{k}\right\|\right) \\
& \geq \frac{\mu}{4\|\mathbf{P}\|}\left\|\mathbf{y}_{e}^{k}\right\|_{\mathbf{P}}=\frac{2 \theta}{\alpha}\left\|\mathbf{y}_{e}^{k}\right\|_{\mathbf{P}} .
\end{aligned}
$$

The second inequality is due to the special form of $\rho_{k}$ in (4.3) and (4.4), and the last inequality is due to the relation

$$
a b \leq \frac{1}{2}\left(2 a^{2}+\frac{1}{2} b^{2}\right) .
$$

The inequality (4.11) is incorporated in the left side of (4.10). We multiply the resulting inequality by $K:=k_{0}+k$, substitute $\rho_{k}=K \theta$, exploit the assumption (4.5) and the inequality $K(K-2) \leq(K-1)^{2}$ to obtain

$$
\begin{aligned}
\alpha K \Delta^{k} \leq & \theta\left((K-1)^{2}\left\|\mathbf{y}_{e}^{k}\right\|_{\mathbf{P}}^{2}-K^{2}\left\|\mathbf{y}_{e}^{k+1}\right\|_{\mathbf{P}}^{2}\right)+\frac{1}{\theta}\left(\left\|\boldsymbol{\lambda}_{e}^{k}\right\|^{2}-\left\|\boldsymbol{\lambda}_{e}^{k+1}\right\|^{2}\right) \\
& +\alpha \sum_{i=1}^{m}\left(\frac{K\left\|\mathbf{x}_{e, i}^{k}\right\|^{2}}{\Gamma_{i}^{k}}-\frac{(K+1)\left\|\mathbf{x}_{e, i}^{k+1}\right\|^{2}}{\Gamma_{i}^{k+1}}\right) .
\end{aligned}
$$


Summing this inequality for $k$ between 1 and $t$, with $K=k_{0}+k$, yields

$$
\alpha \sum_{k=1}^{t}\left(k_{0}+k\right) \Delta^{k}+\left(k_{0}+t\right)^{2} \theta\left\|\mathbf{y}^{t+1}-\mathbf{x}^{*}\right\|_{\mathbf{P}}^{2} \leq \bar{c}
$$

where $\bar{c}$ is defined in (4.9). Substituting for $\Delta^{k}$ using (3.5) and discarding the $\mathbf{y}^{t+1}$ term, we have

$$
\alpha \sum_{k=1}^{t}\left(k_{0}+k\right)\left[\mathcal{L}\left(\mathbf{z}^{k}, \boldsymbol{\lambda}^{*}\right)-\Phi\left(\mathbf{x}^{*}\right)\right] \leq \bar{c} .
$$

The convexity of $\Phi$ and the definition of $\tilde{\mathbf{z}}^{k}$ in (4.8) yield

$$
\left.\mathcal{L}\left(\tilde{\mathbf{z}}^{k}, \boldsymbol{\lambda}^{*}\right)\right) \leq \frac{2}{t(t+1)+2 k_{0} t} \sum_{k=1}^{t}\left(k_{0}+k\right) \mathcal{L}\left(\mathbf{z}^{k}, \boldsymbol{\lambda}^{*}\right),
$$

which together with (4.13) gives (4.6). In addition, since $\Delta^{k} \geq 0$, (4.12) also implies (4.7). $\mathrm{c}$

As noted at the end of Section 2 for either of the parameter choices (2.4) or (2.5), $\gamma^{l} \geq l^{2} \Theta$ for some constant $\Theta>0$, independent of $k$ and $l$. Hence, for $l$ sufficiently large, the requirement (4.5) at iteration $k+1$ is satisfied.

5. Linear Convergence. For the analysis of linear convergence rate of I-ADMM, we assume that $\psi$ has the additional property that $\psi(t) \leq c_{\psi} t$ for all $t \geq 0$, where $c_{\psi}>0$ is a constant. Let us define

$$
e_{i}(\mathbf{y}, \boldsymbol{\lambda})=\left\|\mathbf{y}_{i}-\operatorname{prox}_{h_{i}}\left(\mathbf{y}_{i}-\nabla f_{i}\left(\mathbf{y}_{i}\right)-\mathbf{A}_{i}^{\top} \boldsymbol{\lambda}\right)\right\| .
$$

We begin with the following lemma.

Lemma 5.1. If the parameters $\delta^{l}$ and $\alpha^{l}$ in Algorithm 2.2 are chosen according to either (2.4) or (2.5) and $\psi(t) \leq c_{\psi} t$, then for any $k \geq 2$, we have

$$
\sum_{i=1}^{m} e_{i}\left(\mathbf{y}^{k+1}, \boldsymbol{\lambda}^{k+1}\right) \leq c\left(d_{k}+d_{k-1}\right)
$$

where $c>0$ is a generic constant which only depends on the problem data and algorithm parameters such as $\rho$ and $c_{\psi}$ and

$$
d_{k}=\left\|\mathbf{y}^{k}-\mathbf{z}^{k}\right\|+\left\|\mathbf{A} \mathbf{z}^{k}-\mathbf{b}\right\|+\sqrt{R^{k}} .
$$

Proof. For any $\mathbf{p}_{i}$ and $\mathbf{q}_{i} \in \mathbb{R}^{n_{i}}, i=1,2$, it follows from the triangle inequality and the nonexpansive property of the prox operator that

$$
\begin{aligned}
& \left\|\mathbf{p}_{1}-\operatorname{prox}_{h_{i}}\left(\mathbf{q}_{1}\right)\right\| \\
= & \left\|\left[\mathbf{p}_{2}-\operatorname{prox}_{h_{i}}\left(\mathbf{q}_{2}\right)\right]+\left[\mathbf{p}_{1}-\mathbf{p}_{2}\right]+\left[\operatorname{prox}_{h_{i}}\left(\mathbf{q}_{2}\right)-\operatorname{prox}_{h_{i}}\left(\mathbf{q}_{1}\right)\right]\right\| \\
\leq & \left\|\mathbf{p}_{2}-\operatorname{prox}_{h_{i}}\left(\mathbf{q}_{2}\right)\right\|+\left\|\mathbf{p}_{1}-\mathbf{p}_{2}\right\|+\left\|\mathbf{q}_{1}-\mathbf{q}_{2}\right\| .
\end{aligned}
$$

We identify $\left\|\mathbf{p}_{1}-\operatorname{prox}_{h_{i}}\left(\mathbf{q}_{1}\right)\right\|$ with $e_{i}\left(\mathbf{y}^{k+1}, \boldsymbol{\lambda}^{k+1}\right)$ and $\left\|\mathbf{p}_{2}-\operatorname{prox}_{h_{i}}\left(\mathbf{q}_{2}\right)\right\|$ with $e_{i}\left(\mathbf{z}^{k}, \boldsymbol{\lambda}^{k}\right)$, and use (5.4) to obtain the following bound for $e_{i}\left(\mathbf{y}^{k+1}, \boldsymbol{\lambda}^{k+1}\right)$ in terms of $e_{i}\left(\mathbf{z}^{k}, \boldsymbol{\lambda}^{k}\right)$ :

$$
e_{i}\left(\mathbf{y}^{k+1}, \boldsymbol{\lambda}^{k+1}\right) \leq e_{i}\left(\mathbf{z}^{k}, \boldsymbol{\lambda}^{k}\right)+\left(2+\zeta_{i}\right)\left\|\mathbf{y}_{i}^{k+1}-\mathbf{z}_{i}^{k}\right\|+\left\|\mathbf{A}_{i}^{\top}\left(\boldsymbol{\lambda}^{k+1}-\boldsymbol{\lambda}^{k}\right)\right\|,
$$


where $\zeta_{i}$ is the Lipschitz constant for $\nabla f_{i}$. The update formula for $\boldsymbol{\lambda}^{k+1}$ implies that $\boldsymbol{\lambda}^{k+1}-\boldsymbol{\lambda}^{k}=\alpha \rho\left(\mathbf{A} \mathbf{z}^{k}-\mathbf{b}\right)=\alpha \rho \mathbf{r}_{k}$, where $\mathbf{r}_{k}=\mathbf{A} \mathbf{z}^{k}-\mathbf{b}$. With this substitution, the bound for $e_{i}\left(\mathbf{y}^{k+1}, \boldsymbol{\lambda}_{i}^{k+1}\right)$ becomes

$$
e_{i}\left(\mathbf{y}^{k+1}, \boldsymbol{\lambda}^{k+1}\right) \leq e_{i}\left(\mathbf{z}^{k}, \boldsymbol{\lambda}^{k}\right)+\left(2+\zeta_{i}\right)\left\|\mathbf{y}_{i}^{k+1}-\mathbf{z}_{i}^{k}\right\|+\alpha \rho\left\|\mathbf{A}_{i}^{\top} \mathbf{r}^{k}\right\| .
$$

Let $\nu_{i}>0$ denote the smallest eigenvalue of $\mathbf{Q}_{i}$. The analysis is partitioned into two cases:

Case 1. $\Gamma_{i}^{k}>4 /\left(\rho \nu_{i}\right)$. Again, by property (5.4), we have

$$
e_{i}\left(\mathbf{z}^{k}, \boldsymbol{\lambda}^{k}\right) \leq e_{i}\left(\overline{\mathbf{x}}^{k}, \boldsymbol{\lambda}^{k}\right)+\left(2+\zeta_{i}\right)\left\|\mathbf{z}_{i}^{k}-\overline{\mathbf{x}}_{i}^{k}\right\|,
$$

where $\overline{\mathbf{x}}^{k}$ is given in (3.2). The first-order optimality conditions for $\overline{\mathbf{x}}_{i}^{k}$ can be written

$$
\overline{\mathbf{x}}_{i}^{k}=\operatorname{prox}_{h_{i}}\left(\overline{\mathbf{x}}_{i}^{k}-\nabla f_{i}\left(\overline{\mathbf{x}}_{i}^{k}\right)-\rho \mathbf{A}_{i}^{\top}\left(\mathbf{A}_{i} \mathbf{y}_{i}^{k}-\mathbf{b}_{i}^{k}+\boldsymbol{\lambda}^{k} / \rho\right)-\rho \mathbf{Q}_{i}\left(\overline{\mathbf{x}}_{i}^{k}-\mathbf{y}_{i}^{k}\right)\right) .
$$

Using this formula for the first $\overline{\mathbf{x}}_{i}^{k}$ on the right side of the identity

$$
e_{i}\left(\overline{\mathbf{x}}^{k}, \boldsymbol{\lambda}\right)=\left\|\overline{\mathbf{x}}_{i}^{k}-\operatorname{prox}_{h_{i}}\left(\overline{\mathbf{x}}_{i}^{k}-\nabla f_{i}\left(\overline{\mathbf{x}}_{i}^{k}\right)-\mathbf{A}_{i}^{\top} \boldsymbol{\lambda}\right)\right\|,
$$

along with the nonexpansive property of prox operator, we have

$$
e_{i}\left(\overline{\mathbf{x}}^{k}, \boldsymbol{\lambda}^{k}\right) \leq \rho\left(\left\|\mathbf{A}_{i}^{\top}\left(\mathbf{A}_{i} \mathbf{y}_{i}^{k}-\mathbf{b}_{i}^{k}\right)\right\|+\left\|\mathbf{Q}_{i}\left(\overline{\mathbf{x}}_{i}^{k}-\mathbf{y}_{i}^{k}\right)\right\|\right) .
$$

The definition of $\mathbf{b}_{i}^{k}$ yields

$$
\begin{aligned}
\mathbf{A}_{i} \mathbf{y}_{i}^{k}-\mathbf{b}_{i}^{k} & =\sum_{j<i} \mathbf{A}_{j} \mathbf{z}_{j}^{k}+\sum_{j \geq i} \mathbf{A}_{j} \mathbf{y}_{j}^{k}-\mathbf{b} \\
& =\mathbf{A} \mathbf{z}^{k}-\mathbf{b}+\sum_{j \geq i} \mathbf{A}_{j}\left(\mathbf{y}_{j}^{k}-\mathbf{z}_{j}^{k}\right) \\
& =\mathbf{r}_{k}+\sum_{j \geq i} \mathbf{A}_{j}\left(\mathbf{y}_{j}^{k}-\mathbf{z}_{j}^{k}\right) .
\end{aligned}
$$

It follows that

$$
\left\|\mathbf{A}_{i}^{\top}\left(\mathbf{A}_{i} \mathbf{y}_{i}^{k}-\mathbf{b}_{i}^{k}\right)\right\| \leq c\left(\left\|\mathbf{r}_{k}\right\|+\left\|\mathbf{y}^{k}-\mathbf{z}^{k}\right\|\right),
$$

and

$$
e_{i}\left(\overline{\mathbf{x}}^{k}, \boldsymbol{\lambda}^{k}\right) \leq c\left(\left\|\mathbf{r}_{k}\right\|+\left\|\mathbf{y}^{k}-\mathbf{z}^{k}\right\|+\left\|\overline{\mathbf{x}}_{i}^{k}-\mathbf{z}_{i}^{k}\right\|\right) .
$$

Combining this with (5.6) gives

$$
e_{i}\left(\mathbf{z}^{k}, \boldsymbol{\lambda}^{k}\right) \leq c\left(\left\|\mathbf{r}_{k}\right\|+\left\|\mathbf{y}^{k}-\mathbf{z}^{k}\right\|+\left\|\overline{\mathbf{x}}_{i}^{k}-\mathbf{z}_{i}^{k}\right\|\right) .
$$

Now, by Lemma 3.1 we have

$$
\sqrt{\rho \nu_{i}}\left\|\mathbf{z}_{i}^{k}-\overline{\mathbf{x}}_{i}^{k}\right\| \leq \frac{\left\|\mathbf{x}_{i}^{k}-\overline{\mathbf{x}}_{i}^{k}\right\|}{\sqrt{\Gamma_{i}^{k}}} \leq \frac{\left\|\mathbf{x}_{i}^{k}-\mathbf{z}_{i}^{k}\right\|+\left\|\mathbf{z}_{i}^{k}-\overline{\mathbf{x}}_{i}^{k}\right\|}{\sqrt{\Gamma_{i}^{k}}} .
$$

The stopping condition in Step $1 \mathrm{~b}$ gives

$$
\frac{\left\|\mathbf{x}_{i}^{k}-\mathbf{z}_{i}^{k}\right\|}{\sqrt{\Gamma_{i}^{k}}} \leq \psi\left(\epsilon^{k-1}\right) \leq c \epsilon^{k-1} .
$$


Hence, by (5.9) we have

$$
\left(\frac{-1+\sqrt{\Gamma_{i}^{k} \rho \nu_{i}}}{\sqrt{\Gamma_{i}^{k}}}\right)\left\|\mathbf{z}_{i}^{k}-\overline{\mathbf{x}}_{i}^{k}\right\| \leq \frac{\left\|\mathbf{x}_{i}^{k}-\mathbf{z}_{i}^{k}\right\|}{\sqrt{\Gamma_{i}^{k}}} \leq c \epsilon^{k-1} .
$$

Therefore, the Case 1 condition $\Gamma_{i}^{k}>4 /\left(\rho \nu_{i}\right)$ implies that

$$
\left\|\mathbf{z}_{i}^{k}-\overline{\mathbf{x}}_{i}^{k}\right\| \leq c \epsilon^{k-1}
$$

and by (5.8), we have

$$
e_{i}\left(\mathbf{z}^{k}, \boldsymbol{\lambda}^{k}\right) \leq c\left(\epsilon^{k-1}+\left\|\mathbf{y}^{k}-\mathbf{z}^{k}\right\|+\left\|\mathbf{r}_{k}\right\|\right) .
$$

Case 2. $\Gamma_{i}^{k} \leq 4 /\left(\rho \nu_{i}\right)$. It is shown in [21, pp. 227-228] that when the parameters $\delta^{l}$ and $\alpha^{l}$ are chosen according to either (2.4) or (2.5), there exists a constant $\Theta>0$, independent of $k$ and $l$, such that $\gamma^{l} \geq l^{2} \Theta$. Since the $\gamma^{l}$ are increasing functions of $l$ and $\Gamma_{i}^{k}$ is the final value of $\gamma^{l}$ in Step 1, it follows from the uniform bound on $\Gamma_{i}^{k}$ in Case 2, and the quadratic growth in $\gamma^{l}$, that the final $l$ value in Step 1, which we denote $l_{i}^{k}$, is uniformly bounded as a function of $i$ and $k$. Also, it follows from the quadratic growth of $\gamma^{l}$ and equations (5.18) and (5.20) in [21] that $\delta^{l}$ is uniformly (in $k, l$, and $i$ ) bounded.

By the definition of $\gamma^{l}$ in Algorithm 2.2, we have $\left(1-\alpha^{l}\right) \gamma^{l}=\gamma^{l-1}$, or equivalently, $\alpha^{l} \gamma^{l}=\gamma^{l}-\gamma^{l-1}$ (with the convention that $\gamma^{0}=0$ ). Summing this identity over $l$ yields

$$
\gamma^{l}=\sum_{j=1}^{l} \alpha^{j} \gamma^{j}
$$

Next, we multiply the definition $\mathbf{a}_{i k}^{j}=\left(1-\alpha^{j}\right) \mathbf{a}_{i k}^{j-1}+\alpha^{j} \mathbf{u}_{i k}^{j}$ by $\gamma^{j}$ and sum over $j$ between 1 and $l$. Again, exploiting the identity $\left(1-\alpha^{j}\right) \gamma^{j}=\gamma^{j-1}$ yields

$$
\mathbf{a}_{i k}^{l}=\frac{1}{\gamma^{l}} \sum_{j=1}^{l}\left(\gamma^{j} \alpha^{j}\right) \mathbf{u}_{i k}^{j} .
$$

It follows from (5.12), that $\mathbf{a}_{i k}^{l}$ is a convex combination of $\mathbf{u}_{i k}^{j}, 1 \leq j \leq l$. If $p_{i k}^{j} \in[0,1]$ denotes the coefficients in the convex combination, we have

$$
\mathbf{a}_{i k}^{l}=\sum_{j=1}^{l} p_{i k}^{j} \mathbf{u}_{i k}^{j},
$$

Since $\mathbf{z}_{i}^{k}=\mathbf{a}_{i k}^{L}$ for $L=l_{i}^{k}$, Jensen's inequality gives

$$
\begin{aligned}
e_{i}\left(\mathbf{z}^{k}, \boldsymbol{\lambda}^{k}\right) & \leq \sum_{l=1}^{l_{i}^{k}} p_{i k}^{l}\left\|\mathbf{u}_{i k}^{l}-\operatorname{prox}_{h_{i}}\left(\mathbf{z}_{i}^{k}-\nabla f_{i}\left(\mathbf{z}_{i}^{k}\right)-\mathbf{A}_{i}^{\top} \boldsymbol{\lambda}^{k}\right)\right\| \\
& \leq \sum_{l=1}^{l_{i}^{k}}\left\|\mathbf{u}_{i k}^{l}-\operatorname{prox}_{h_{i}}\left(\mathbf{z}_{i}^{k}-\nabla f_{i}\left(\mathbf{z}_{i}^{k}\right)-\mathbf{A}_{i}^{\top} \boldsymbol{\lambda}^{k}\right)\right\| .
\end{aligned}
$$

Now, by the formula for $\mathbf{u}_{i k}^{l}$ in Alg. 2.2. we have $\mathbf{u}_{i k}^{l}=\operatorname{prox}_{h_{i}}\left(\mathbf{q}_{2}\right)$, where $\mathbf{q}_{2}=\mathbf{u}_{i k}^{l}-\nabla f_{i}\left(\overline{\mathbf{a}}_{i k}^{l}\right)-\delta_{i k}^{l}\left(\mathbf{u}_{i k}^{l}-\mathbf{u}_{i k}^{l-1}\right)-\rho \mathbf{A}_{i}^{\top}\left(\mathbf{A}_{i} \mathbf{y}_{i}^{k}-\mathbf{b}_{i}^{k}+\lambda^{k} / \rho\right)-\rho \mathbf{Q}_{i}\left(\mathbf{u}_{i k}^{l}-\mathbf{y}_{i}^{k}\right)$. 
We utilize (5.4) with $\mathbf{q}_{1}=\mathbf{z}_{i}^{k}-\nabla f_{i}\left(\mathbf{z}_{i}^{k}\right)-\mathbf{A}_{i}^{\top} \boldsymbol{\lambda}^{k}$, with $\mathbf{q}_{2}$ as given above, and with $\mathbf{p}_{1}=\mathbf{p}_{2}=\mathbf{u}_{i k}^{l}$. Hence, $\mathbf{p}_{2}-\operatorname{prox}_{h_{i}}\left(\mathbf{q}_{2}\right)=\mathbf{0}$ and by (5.4), it follows that

$$
\begin{gathered}
c\left(\left\|\mathbf{u}_{i k}^{l}-\mathbf{z}_{i}^{k}\right\|+\left\|\overline{\mathbf{a}}_{i k}^{l}-\mathbf{z}_{i}^{k}\right\|+\left\|\mathbf{u}_{i k}^{l}-\mathbf{u}_{i k}^{l-1}\right\|+\left\|\mathbf{A}_{i}^{\top}\left(\mathbf{A}_{i} \mathbf{y}_{i}^{k}-\mathbf{b}_{i}^{k}\right)\right\|+\left\|\mathbf{u}_{i k}^{l}-\mathbf{y}_{i}^{k}\right\|\right) \leq \\
c\left(\left\|\mathbf{u}_{i k}^{l}-\mathbf{z}_{i}^{k}\right\|+\left\|\overline{\mathbf{a}}_{i k}^{l}-\mathbf{z}_{i}^{k}\right\|+\left\|\mathbf{u}_{i k}^{l}-\mathbf{u}_{i k}^{l-1}\right\|+\left\|\mathbf{A}_{i}^{\top}\left(\mathbf{A}_{i} \mathbf{y}_{i}^{k}-\mathbf{b}_{i}^{k}\right)\right\|+\left\|\mathbf{y}_{i}^{k}-\mathbf{z}_{i}^{k}\right\|\right)
\end{gathered}
$$

Each of the terms on the right side of (5.16) is now analyzed.

Based on (5.7), the trailing two terms in (5.16) have the bound

$$
\left\|\mathbf{A}_{i}^{\top}\left(\mathbf{A}_{i} \mathbf{y}_{i}^{k}-\mathbf{b}_{i}^{k}\right)\right\|+\left\|\mathbf{y}_{i}^{k}-\mathbf{z}_{i}^{k}\right\| \leq c\left(\left\|\mathbf{r}_{k}\right\|+\left\|\mathbf{y}^{k}-\mathbf{z}^{k}\right\|\right) .
$$

The remaining terms in (5.16) are bounded by $c \sqrt{r_{i}^{k}}$ as will now be shown. The bound $\left\|\mathbf{u}_{i k}^{l}-\mathbf{u}_{i k}^{l-1}\right\| \leq c \sqrt{r_{i}^{k}}$ is a trivial consequence of the definition of $r_{i}^{k}$ and the uniform bound on $\Gamma_{i}^{k}$ in Case 2. By the definition $\overline{\mathbf{a}}_{i k}^{l}=\left(1-\alpha^{l}\right)\left(\mathbf{a}_{i k}^{l-1}-\mathbf{u}_{i k}^{l-1}\right)+\mathbf{u}_{i k}^{l-1}$, it follows that

$$
\left\|\overline{\mathbf{a}}_{i k}^{l}-\mathbf{z}_{i}^{k}\right\| \leq\left\|\mathbf{a}_{i k}^{l-1}-\mathbf{u}_{i k}^{l-1}\right\|+\left\|\mathbf{u}_{i k}^{l-1}-\mathbf{z}_{i}^{k}\right\| .
$$

This inequality and the fact that $\mathbf{z}_{i}^{k}=\mathbf{a}_{i k}^{l}$ for $l=l_{i}^{k}$ implies that all the remaining terms in (5.16) have the form $\left\|\mathbf{a}_{i k}^{l}-\mathbf{u}_{i k}^{t}\right\|$ for some $l \in\left[1, l_{i}^{k}\right]$ and some $t \in[1, l]$. Combine (5.14), Jensen's inequality, the fact that $l \leq l_{i}^{k}$ where $l_{i}^{k}$ is uniformly bounded in Case 2, and the Schwarz inequality to obtain

$$
\left\|\mathbf{a}_{i k}^{l}-\mathbf{u}_{i k}^{t}\right\| \leq \sum_{j=1}^{l}\left\|\mathbf{u}_{i k}^{j}-\mathbf{u}_{i k}^{t}\right\| \leq l \sum_{j=1}^{l}\left\|\mathbf{u}_{i k}^{j}-\mathbf{u}_{i k}^{j-1}\right\| \leq c \sqrt{r_{i}^{k}},
$$

These bounds for the terms in (5.16) combine to yield

$$
\left\|\mathbf{u}_{i k}^{l}-\operatorname{prox}_{h_{i}}\left(\mathbf{z}_{i}^{k}-\nabla f_{i}\left(\mathbf{z}_{i}^{k}\right)-\mathbf{A}_{i}^{\top} \boldsymbol{\lambda}^{k}\right)\right\| \leq c\left(\left\|\mathbf{r}_{k}\right\|+\left\|\mathbf{y}^{k}-\mathbf{z}^{k}\right\|+\sqrt{r_{i}^{k}}\right) .
$$

Moreover, by (5.15) and the Case 2 uniform bound on $l_{i}^{k}$, we have

$$
e_{i}\left(\mathbf{z}^{k}, \boldsymbol{\lambda}^{k}\right) \leq c\left(\left\|\mathbf{r}_{k}\right\|+\left\|\mathbf{y}^{k}-\mathbf{z}^{k}\right\|+\sqrt{r_{i}^{k}}\right) .
$$

Combine this with the Case 1 lower bound (5.11) gives

$$
e_{i}\left(\mathbf{z}^{k}, \boldsymbol{\lambda}^{k}\right) \leq c\left(\epsilon^{k-1}+\left\|\mathbf{r}_{k}\right\|+\left\|\mathbf{y}^{k}-\mathbf{z}^{k}\right\|+\sqrt{r_{i}^{k}}\right) .
$$

Inserting this in (5.5) yields

$$
e_{i}\left(\mathbf{y}^{k+1}, \boldsymbol{\lambda}^{k+1}\right) \leq c\left(\epsilon^{k-1}+\left\|\mathbf{r}_{k}\right\|+\left\|\mathbf{y}^{k}-\mathbf{z}^{k}\right\|+\sqrt{r_{i}^{k}}+\left\|\mathbf{y}^{k+1}-\mathbf{y}^{k}\right\|\right) .
$$

Based on the back substitution formula $\mathbf{y}^{k+1}-\mathbf{y}^{k}=\alpha \mathbf{M}^{-\top} \mathbf{Q}\left(\mathbf{z}^{k}-\mathbf{y}^{k}\right)$, this reduces to

$$
e_{i}\left(\mathbf{y}^{k+1}, \boldsymbol{\lambda}^{k+1}\right) \leq c\left(\epsilon^{k-1}+\left\|\mathbf{r}_{k}\right\|+\left\|\mathbf{y}^{k}-\mathbf{z}^{k}\right\|+\sqrt{r_{i}^{k}}\right) .
$$


Since $\epsilon^{k-1} \leq c d_{k-1}$ and $\left\|\mathbf{r}_{k}\right\|+\left\|\mathbf{y}^{k}-\mathbf{z}^{k}\right\|+\sqrt{r_{i}^{k}} \leq d_{k}$, the proof is complete.

The expression $E_{k}$ defined in (3.3) measures the energy between the current iterate $\left(\mathbf{x}_{k}, \mathbf{y}_{k}, \boldsymbol{\lambda}_{k}\right)$ and a given $\left(\mathbf{x}^{*}, \mathbf{x}^{*}, \boldsymbol{\lambda}^{*}\right)$. Let $E_{k}^{*}$ denote the minimum energy between the iterate and all possible $\left(\mathbf{x}^{*}, \boldsymbol{\lambda}^{*}\right) \in \mathcal{W}^{*}$. We will show that when an error bound condition holds, there exists a constant $\kappa<1$ such that $E_{k+2}^{*} \leq \kappa E_{k}^{*}$.

The error bound condition relates the KKT error to the Euclidean distance to $\mathcal{W}^{*}$. The KKT error $K$ is given by

$$
K(\mathbf{x}, \boldsymbol{\lambda})=\|\mathbf{A} \mathbf{x}-\mathbf{b}\|+\sum_{i=1}^{m} e_{i}(\mathbf{x}, \boldsymbol{\lambda})
$$

When $K(\mathbf{x}, \boldsymbol{\lambda})=0$, the first-order optimality conditions hold. The Euclidean distance from $(\mathbf{x}, \boldsymbol{\lambda})$ to $\mathcal{W}^{*}$ will be measured by

$$
\mathcal{E}(\mathbf{x}, \boldsymbol{\lambda})=\min \left\{\rho\left\|\mathbf{x}-\mathbf{x}^{*}\right\|_{\mathbf{P}}^{2}+\frac{1}{\rho}\left\|\boldsymbol{\lambda}-\boldsymbol{\lambda}^{*}\right\|^{2}:\left(\mathbf{x}^{*}, \boldsymbol{\lambda}^{*}\right) \in \mathcal{W}^{*}\right\}^{1 / 2} .
$$

Note that $\mathbf{P}=\mathbf{M Q}^{-1} \mathbf{M}^{\top}$ is positive definite since $\mathbf{M}$ is invertible. Also, by [1, Prop. 6.1.2], every solution of (1.1) has exactly the same set of Lagrange multipliers. If $\mathbf{X}^{*}$ and $\boldsymbol{\Lambda}^{*}$ denote the set of solutions and multipliers for (1.1), then $\mathcal{W}^{*}=\mathbf{X}^{*} \times \boldsymbol{\Lambda}^{*}$ is a closed, convex set, and there exists a unique $(\tilde{\mathbf{x}}, \tilde{\boldsymbol{\lambda}}) \in \mathcal{W}^{*}$ that achieves the minimum in (5.19). The local error bound assumption is as follows:

Assumption 5.1. There exist constants $\beta>0$ and $\eta>0$ such that $\mathcal{E}(\mathbf{x}, \boldsymbol{\lambda}) \leq$ $\eta K(\mathbf{x}, \boldsymbol{\lambda})$ whenever $\mathcal{E}(\mathbf{x}, \boldsymbol{\lambda}) \leq \beta$.

The local error bound condition is equivalent to saying that in a neighborhood of $\mathcal{W}^{*}$, the Euclidean distance to $\mathcal{W}^{*}$ is bound by the KKT error, which is often used to analyze the linear convergence behavior of an optimization algorithm. More recently, a partial error bound condition based on the ADMM iterates instead of conditions on the optimization problem is proposed in 34. Under such conditions, linear convergence is also established for a 2-block ADMM.

A multivalued mapping $F$ is piecewise polyhedral if its graph Gph $F:=\{(\mathbf{x}, \mathbf{y})$ : $\mathbf{y} \in F(\mathbf{x})\}$ is a union of finitely many polyhedral sets. The local error bound condition (Assumption 5.1) holds when $\nabla f_{i}$ is affine and $\partial h_{i}$ is piecewise polyhedral for $i=1, \ldots, m$ [23, 36, 42. Note that when $(\mathbf{x}, \boldsymbol{\lambda})$ is restricted to a bounded set, the requirement that $\mathcal{E}(\mathbf{x}, \boldsymbol{\lambda}) \leq \beta$ can be dropped. That is, when $\mathcal{E}(\mathbf{x}, \boldsymbol{\lambda})>\beta$, $K(\mathbf{x}, \boldsymbol{\lambda})$ is strictly positive, and by taking the constant $\eta$ large enough, the bound $\mathcal{E}(\mathbf{x}, \boldsymbol{\lambda}) \leq \eta K(\mathbf{x}, \boldsymbol{\lambda})$ holds over the entire set. In our analysis, the error bound condition is applied to the iterates $\left(\mathbf{y}^{k}, \lambda^{k}\right)$ which lie in a bounded set by Lemma 3.2 so the requirement that $\mathcal{E}(\mathbf{x}, \boldsymbol{\lambda}) \leq \beta$ is unnecessary.

THEOREM 5.2. If the parameters $\delta^{l}$ and $\alpha^{l}$ in Algorithm 2.2 are chosen according to either (2.4) or (2.5), $\psi(t) \leq c_{\psi} t$, and Assumption 5.1 holds, then there exists $\kappa<1$ such that $E_{k+2}^{*} \leq \kappa E_{k}^{*}$ at every iteration of Algorithm 2.1.

Proof. Let $\left(\tilde{\mathbf{y}}^{k+1}, \tilde{\boldsymbol{\lambda}}^{k+1}\right) \in \mathcal{W}^{*}$ be the unique minimizer in (5.19) corresponding to $(\mathbf{x}, \boldsymbol{\lambda})=\left(\mathbf{y}^{k+1}, \boldsymbol{\lambda}^{k+1}\right)$. By the stopping condition in Step 1b of Algorithm 2.2. and the definition of $\Gamma_{i}^{k}$ in Step 1c, the sequence $\Gamma_{i}^{k}$ is nondecreasing in $k$ by Remark 2.1 Since $\Gamma_{i}^{k}$ is nondecreasing in $k$, it follows from the triangle inequality and the back substitution formula $\mathbf{y}^{k+1}-\mathbf{y}^{k}=\alpha \mathbf{M}^{-\top} \mathbf{Q}\left(\mathbf{z}^{k}-\mathbf{y}^{k}\right)$ that for any $i \in[1, m]$, we have

$$
\frac{\left\|\mathbf{x}_{i}^{k+1}-\tilde{\mathbf{y}}_{i}^{k+1}\right\|}{\sqrt{\Gamma_{i}^{k+1}}} \leq \frac{\left\|\mathbf{x}_{i}^{k+1}-\mathbf{z}_{i}^{k}\right\|+\left\|\mathbf{z}_{i}^{k}-\mathbf{y}_{i}^{k}\right\|+\left\|\mathbf{y}_{i}^{k}-\mathbf{y}_{i}^{k+1}\right\|+\left\|\mathbf{y}_{i}^{k+1}-\tilde{\mathbf{y}}_{i}^{k+1}\right\|}{\sqrt{\Gamma_{i}^{k+1}}}
$$




$$
\begin{aligned}
& \leq \frac{\left\|\mathbf{x}_{i}^{k+1}-\mathbf{z}_{i}^{k}\right\|}{\sqrt{\Gamma_{i}^{k}}}+\frac{\left\|\mathbf{z}_{i}^{k}-\mathbf{y}_{i}^{k}\right\|+\left\|\mathbf{y}_{i}^{k}-\mathbf{y}_{i}^{k+1}\right\|+\left\|\mathbf{y}_{i}^{k+1}-\tilde{\mathbf{y}}_{i}^{k+1}\right\|}{\sqrt{\Gamma_{i}^{1}}} \\
& \leq \frac{\left\|\mathbf{x}_{i}^{k+1}-\mathbf{z}_{i}^{k}\right\|}{\sqrt{\Gamma_{i}^{k}}}+c\left(\left\|\mathbf{z}^{k}-\mathbf{y}^{k}\right\|+\left\|\mathbf{y}_{i}^{k+1}-\tilde{\mathbf{y}}_{i}^{k+1}\right\|\right),
\end{aligned}
$$

where $c>0$ denotes a generic constant, independent of $k$.

As noted earlier, when the parameters $\delta^{l}$ and $\alpha^{l}$ in Algorithm 2.2 are chosen according to either (2.4) or (2.5), we have $\xi^{l}=\delta^{l} \alpha^{l} \gamma^{l}=1$. By equation (2.7) with $L=l_{i}^{k}, \mathbf{u}=\mathbf{a}_{i}^{L}=\mathbf{z}_{i}^{k}, \mathbf{u}_{i}^{L}=\mathbf{x}^{k+1}$, and $\mathbf{u}_{i}^{0}=\mathbf{x}_{k}$, we obtain the relation

$$
\frac{\left\|\mathbf{z}_{i}^{k}-\mathbf{x}_{i}^{k+1}\right\|}{\sqrt{\Gamma_{i}^{k}}} \leq \frac{\left\|\mathbf{z}_{i}^{k}-\mathbf{x}_{i}^{k}\right\|}{\sqrt{\Gamma_{i}^{k}}} \leq \psi\left(\epsilon^{k-1}\right),
$$

where the last inequality is due to the stopping condition in Step 1b. Combining this with (5.20) yields

$$
\frac{\left\|\mathbf{x}_{i}^{k+1}-\tilde{\mathbf{y}}_{i}^{k+1}\right\|}{\sqrt{\Gamma_{i}^{k+1}}} \leq \psi\left(\epsilon^{k-1}\right)+c\left(\left\|\mathbf{z}^{k}-\mathbf{y}^{k}\right\|+\left\|\mathbf{y}_{i}^{k+1}-\tilde{\mathbf{y}}_{i}^{k+1}\right\|\right) .
$$

Exploiting the error bound condition, we have

$$
\begin{aligned}
\left\|\mathbf{y}^{k+1}-\tilde{\mathbf{y}}^{k+1}\right\|^{2} & \leq \sqrt{\left\|\mathbf{P}^{-1}\right\|}\left\|\mathbf{y}^{k+1}-\tilde{\mathbf{y}}^{k+1}\right\|_{\mathbf{P}} \\
& \leq c \mathcal{E}\left(\mathbf{y}^{k+1}, \boldsymbol{\lambda}^{k+1}\right) \leq c K\left(\mathbf{y}^{k+1}, \boldsymbol{\lambda}^{k+1}\right) .
\end{aligned}
$$

The constraint violation term in $K$ is estimated as follows:

$$
\left\|\mathbf{A} \mathbf{y}^{k+1}-\mathbf{b}\right\| \leq\|\mathbf{A}\|\left(\left\|\mathbf{y}^{k+1}-\mathbf{y}^{k}\right\|+\left\|\mathbf{y}^{k}-\mathbf{z}^{k}\right\|\right)+\left\|\mathbf{A} \mathbf{z}^{k}-\mathbf{b}\right\| \leq c d_{k},
$$

where the last inequality is due to the back substitution formula and the definition (5.3) of $d_{k}$. Hence, Lemma 5.1 yields

$$
K\left(\mathbf{y}^{k+1}, \boldsymbol{\lambda}^{k+1}\right) \leq c\left(d_{k}+d_{k-1}\right) .
$$

Combine (5.21)-(5.23) to obtain

$$
\frac{\left\|\mathbf{x}_{i}^{k+1}-\tilde{\mathbf{y}}_{i}^{k+1}\right\|}{\sqrt{\Gamma_{i}^{k+1}}} \leq \psi\left(\epsilon^{k-1}\right)+c\left(d_{k}+d_{k-1}\right) \leq c\left(d_{k}+d_{k-1}\right)
$$

since $\psi(t) \leq c_{\psi} t$ and $\epsilon^{k-1} \leq c d_{k-1}$. Since the energy $E_{k+1}^{*}$ corresponds to the minimum of $E_{k+1}$ over all $\left(\mathbf{x}^{*}, \lambda^{*}\right) \in \mathcal{W}^{*}$ and since $\left(\tilde{\mathbf{y}}^{k+1}, \tilde{\boldsymbol{\lambda}}^{k+1}\right) \in \mathcal{W}^{*}$, it follows that

$$
E_{k+1}^{*} \leq \rho\left\|\mathbf{y}^{k+1}-\tilde{\mathbf{y}}^{k+1}\right\|_{\mathbf{P}}^{2}+\frac{1}{\rho}\left\|\boldsymbol{\lambda}^{k+1}-\tilde{\boldsymbol{\lambda}}^{k+1}\right\|^{2}+\alpha \sum_{i=1}^{m} \frac{\left\|\mathbf{x}_{i}^{k+1}-\tilde{\mathbf{y}}_{i}^{k+1}\right\|^{2}}{\Gamma_{i}^{k+1}} .
$$

The first two terms on the right are $\mathcal{E}^{2}\left(\mathbf{y}^{k+1}, \boldsymbol{\lambda}^{k+1}\right)$, while the last term in bounded by (5.24). We have

$$
E_{k+1}^{*} \leq \mathcal{E}^{2}\left(\mathbf{y}^{k+1}, \boldsymbol{\lambda}^{k+1}\right)+c\left(d_{k}+d_{k-1}\right)^{2} .
$$


Combine this with the error bound condition and (5.23) gives

$$
E_{k+1}^{*} \leq c\left(d_{k}+d_{k-1}\right)^{2} .
$$

Suppose that $\left(\hat{\mathbf{x}}^{k}, \hat{\boldsymbol{\lambda}}^{k}\right) \in \mathcal{W}^{*}$ is the unique minimizing $\left(\mathbf{x}^{*}, \boldsymbol{\lambda}^{*}\right) \in \mathcal{W}^{*}$ associated with $E_{k}^{*}$. By Lemma 3.2 and the fact that $\left(\hat{\mathbf{x}}^{k}, \hat{\boldsymbol{\lambda}}^{k}\right) \in \mathcal{W}^{*}$, we have

$$
\begin{gathered}
E_{k}^{*} \geq \rho\left\|\mathbf{y}^{k+1}-\hat{\mathbf{x}}^{k}\right\|_{\mathbf{P}}^{2}+\frac{1}{\rho}\left\|\boldsymbol{\lambda}^{k+1}-\hat{\boldsymbol{\lambda}}^{k}\right\|^{2}+\alpha \sum_{i=1}^{m} \frac{\left\|\mathbf{x}_{i}^{k+1}-\hat{\mathbf{x}}_{i}^{k}\right\|^{2}}{\Gamma_{i}^{k}} \\
+\rho \alpha(1-\alpha)\left(\left\|\mathbf{y}^{k}-\mathbf{z}^{k}\right\|_{\mathbf{Q}}^{2}+\left\|\mathbf{A} \mathbf{z}^{k}-\mathbf{b}\right\|^{2}\right)+\sigma \alpha \sum_{i=1}^{m} R^{k}
\end{gathered}
$$

The first three terms on the right side are bounded from below by $E_{k+1}^{*}$, while the last three terms are bounded from below by $c d_{k}^{2}$ by the definition of $d_{k}$ in (5.3). Hence,

$$
E_{k}^{*} \geq E_{k+1}^{*}+c d_{k}^{2} \text {. }
$$

We replace $k$ by $k-1$ and then use again (5.26) followed by (5.25) to obtain

$$
E_{k-1}^{*} \geq E_{k}^{*}+c d_{k-1}^{2} \geq E_{k+1}^{*}+c\left(d_{k}^{2}+d_{k-1}^{2}\right) \geq(1+c) E_{k+1}^{*},
$$

which completes the proof.

Another linear convergence result is established when the objective $\Phi$ is strongly convex, in which case the solution $\mathrm{x}^{*}$ of (1.1) is unique. Our assumption is the following:

Assumption 5.2. The objective $\Phi$ is strongly convex with modulus $\mu>0$ and there exist constants $\beta>0$ and $\eta>0$ such that

$$
\|\boldsymbol{\lambda}-\tilde{\boldsymbol{\lambda}}\| \leq \eta \sum_{i=1}^{m}\left\|e_{i}\left(\mathbf{x}^{*}, \boldsymbol{\lambda}\right)\right\|
$$

whenever $\|\boldsymbol{\lambda}-\tilde{\boldsymbol{\lambda}}\| \leq \beta$.

The local error bound condition (5.27) holds when $\partial h_{i}$ is piecewise polyhedral for $i=1, \ldots, m[23,36$, 42]. Similar to the comment before Theorem [5.2, the requirement that $\|\boldsymbol{\lambda}-\tilde{\boldsymbol{\lambda}}\| \leq \beta$ can be dropped since it is applied to the iterates $\boldsymbol{\lambda}^{k}$ which lie in a bounded set by Lemma 3.2 .

TheOREM 5.3. If the parameters $\delta^{l}$ and $\alpha^{l}$ in Algorithm 2.2 are chosen according to either (2.4) or (2.5), $\psi(t) \leq c_{\psi}$ t, and Assumption 5.2 holds, then there exists $\kappa<1$ such that $E_{k+2}^{*} \leq \kappa E_{k}^{*}$ at every iteration of Algorithm 2.1.

Proof. By the local error bound condition and by (5.4) with $\mathbf{p}_{1}-\operatorname{prox}_{h_{i}}\left(\mathbf{q}_{1}\right)$ identified with $e_{i}\left(\mathbf{x}^{*}, \boldsymbol{\lambda}^{k+1}\right)$ and $\mathbf{p}_{2}-\operatorname{prox}_{h_{i}}\left(\mathbf{q}_{2}\right)$ identified with $e_{i}\left(\mathbf{z}^{k}, \boldsymbol{\lambda}^{k}\right)$, we have

$$
\begin{aligned}
\left\|\boldsymbol{\lambda}^{k+1}-\tilde{\boldsymbol{\lambda}}^{k+1}\right\| & \leq \eta \sum_{i=1}^{m} e_{i}\left(\mathbf{x}^{*}, \boldsymbol{\lambda}^{k+1}\right) \\
& \leq c\left(\left\|\mathbf{z}^{k}-\mathbf{x}^{*}\right\|+\left\|\boldsymbol{\lambda}^{k+1}-\boldsymbol{\lambda}^{k}\right\|+\sum_{i=1}^{m} e_{i}\left(\mathbf{z}^{k}, \boldsymbol{\lambda}^{k}\right)\right),
\end{aligned}
$$


where $c>0$ is a constant. In the later proof, we again use $c>0$ as a generic constant. By (5.17), it follows that

$$
\sum_{i=1}^{m} e_{i}\left(\mathbf{z}^{k}, \boldsymbol{\lambda}^{k}\right) \leq c\left(\epsilon^{k-1}+\left\|\mathbf{r}_{k}\right\|+\left\|\mathbf{y}^{k}-\mathbf{z}^{k}\right\|+\sqrt{R^{k}}\right) .
$$

Inserting this in (5.28) and recalling that $\boldsymbol{\lambda}^{k+1}-\boldsymbol{\lambda}^{k}=\alpha \rho\left(\mathbf{A} \mathbf{z}^{k}-\mathbf{b}\right)=\alpha \rho \mathbf{r}_{k}$, we have

$$
\left\|\boldsymbol{\lambda}^{k+1}-\tilde{\boldsymbol{\lambda}}^{k+1}\right\| \leq c\left(\epsilon^{k-1}+\left\|\mathbf{z}^{k}-\mathbf{x}^{*}\right\|+\left\|\mathbf{r}_{k}\right\|+\left\|\mathbf{y}^{k}-\mathbf{z}^{k}\right\|+\sqrt{R^{k}}\right) .
$$

Since $\epsilon^{k-1} \leq c d_{k-1}$ and $\left\|\mathbf{r}_{k}\right\|+\left\|\mathbf{y}^{k}-\mathbf{z}^{k}\right\|+\sqrt{R^{k}} \leq d_{k}$, it follows that

$$
\left\|\boldsymbol{\lambda}^{k+1}-\tilde{\boldsymbol{\lambda}}^{k+1}\right\| \leq c\left(d_{k}+d_{k-1}+\left\|\mathbf{z}^{k}-\mathbf{x}^{*}\right\|\right) \text {. }
$$

By (5.21) with $\tilde{\mathbf{y}}^{k+1}=\mathbf{x}^{*}$, we have

$$
\frac{\left\|\mathbf{x}_{i}^{k+1}-\mathbf{x}_{i}^{*}\right\|}{\sqrt{\Gamma_{i}^{k+1}}} \leq c\left(\epsilon^{k-1}+\left\|\mathbf{z}^{k}-\mathbf{y}^{k}\right\|+\left\|\mathbf{y}^{k+1}-\mathbf{x}^{*}\right\|\right) .
$$

The triangle inequality and the back substitution formula yield

$$
\begin{aligned}
\left\|\mathbf{y}^{k+1}-\mathbf{x}^{*}\right\| & \leq\left\|\mathbf{y}^{k+1}-\mathbf{y}^{k}\right\|+\left\|\mathbf{y}^{k}-\mathbf{z}^{k}\right\|+\left\|\mathbf{z}^{k}-\mathbf{x}^{*}\right\| \\
& \leq c\left\|\mathbf{y}^{k}-\mathbf{z}^{k}\right\|+\left\|\mathbf{z}^{k}-\mathbf{x}^{*}\right\| .
\end{aligned}
$$

The bounds $\epsilon^{k-1} \leq c d_{k-1}$ and $\left\|\mathbf{y}^{k}-\mathbf{z}^{k}\right\| \leq d_{k}$ in (5.31) and (5.30) give

$$
\left\|\mathbf{y}^{k+1}-\mathbf{x}^{*}\right\| \leq c d_{k}+\left\|\mathbf{z}^{k}-\mathbf{x}^{*}\right\| \text { and } \frac{\left\|\mathbf{x}_{i}^{k+1}-\mathbf{x}_{i}^{*}\right\|}{\sqrt{\Gamma_{i}^{k+1}}} \leq c\left(d_{k-1}+d_{k}+\left\|\mathbf{z}^{k}-\mathbf{x}^{*}\right\|\right) .
$$

Combine (5.29) and (5.32) to obtain

$$
\begin{aligned}
E_{k+1}^{*} & =\rho\left\|\mathbf{y}^{k+1}-\mathbf{x}^{*}\right\|_{\mathbf{P}}^{2}+\frac{1}{\rho}\left\|\boldsymbol{\lambda}^{k+1}-\tilde{\boldsymbol{\lambda}}^{k+1}\right\|^{2}+\alpha \sum_{i=1}^{m} \frac{\left\|\mathbf{x}_{i}^{k+1}-\mathbf{x}_{i}^{*}\right\|^{2}}{\Gamma_{i}^{k+1}} \\
& \leq c\left(d_{k}+d_{k-1}+\left\|\mathbf{z}^{k}-\mathbf{x}^{*}\right\|\right)^{2} .
\end{aligned}
$$

On the other hand, by Lemma 3.2 and the fact that $\left(\mathrm{x}^{*}, \tilde{\boldsymbol{\lambda}}^{k}\right) \in \mathcal{W}^{*}$, we have

$$
\begin{aligned}
E_{k}^{*} \geq & \rho\left\|\mathbf{y}^{k+1}-\mathbf{x}^{*}\right\|_{\mathbf{P}}^{2}+\frac{1}{\rho}\left\|\mathbf{\lambda}^{k+1}-\tilde{\boldsymbol{\lambda}}^{k}\right\|^{2}+\alpha \sum_{i=1}^{m} \frac{\left\|\mathbf{x}_{i}^{k+1}-\mathbf{x}_{i}^{*}\right\|^{2}}{\Gamma_{i}^{k}} \\
& +\rho \alpha(1-\alpha)\left(\left\|\mathbf{y}^{k}-\mathbf{z}^{k}\right\|_{\mathbf{Q}}^{2}+\left\|\mathbf{A} \mathbf{z}^{k}-\mathbf{b}\right\|^{2}\right)+\sigma \alpha R^{k}+2 \alpha \Delta^{k} \\
\geq & E_{k+1}^{*}+c d_{k}^{2}+\mu\left\|\mathbf{z}^{k}-\mathbf{x}^{*}\right\|^{2},
\end{aligned}
$$

where the last inequality is due to the definition (5.3) of $d_{k}$ and the strong convexity of $\Phi$ :

$$
\Delta^{k}:=\Phi\left(\mathbf{z}^{k}\right)-\Phi\left(\mathbf{x}^{*}\right)+\left(\tilde{\boldsymbol{\lambda}}^{k}, \mathbf{A} \mathbf{z}^{k}-\mathbf{b}\right) \geq \frac{\mu}{2}\left\|\mathbf{z}^{k}-\mathbf{x}^{*}\right\|^{2} .
$$

Finally, we replace $k$ by $k-1$ in (5.34), and then use again (5.34) followed by (5.33) to obtain

$$
E_{k-1}^{*} \geq E_{k}^{*}+c d_{k-1}^{2} \geq E_{k+1}^{*}+c\left(d_{k}^{2}+d_{k-1}^{2}\right)+\mu\left\|\mathbf{z}^{k}-\mathbf{x}^{*}\right\|^{2} \geq(1+c) E_{k+1}^{*},
$$

which completes the proof. $\square$ 
6. Numerical Experiments. In this section, we compare the performance of I-ADMM to that of two different algorithms: (a) linearized ADMM with one linearization step for each subproblem and (b) exact ADMM where the subproblems are solved either by the conjugate gradient method or by an explicit formula. The conjugate gradient method was well suited for the quadratic subproblems in our test set. We tried using a small number of conjugate gradient iterations to solve a subproblem, such as 5 iterations starting from the solution computed in the previous iteration, but found that the scheme did not converge. Instead we continued the CG iteration until the norm of the gradient was at most $10^{-6}$. The one-step ADMM algorithm that we used in (a) for the experiments was the generalized BOSVS algorithm from [21]. This algorithm is globally convergent, and although the penalty term was not linearized, it was possible to quickly solve the subproblems that arise in the imaging test problems using a fast Fourier transform, as explained in [10.

The problems in our experiments were the same image reconstruction problems used in 21]. One image employs a blurred version of the well-known Cameraman image of size $256 \times 256$, while the second set of test problems, which arise in partially parallel imaging (PPI), are found in [10. The observed PPI data, corresponding to 3 different images, are denoted data 1 , data 2 , and data 3 . These image reconstruction problem can be formulated as

$$
\min _{\mathbf{u}} \frac{1}{2}\|\mathbf{F u}-\mathbf{f}\|^{2}+\alpha\|\mathbf{u}\|_{T V}+\beta\left\|\Psi^{\top} \mathbf{u}\right\|_{1},
$$

where $\mathbf{f}$ is the given image data, $\mathbf{F}$ is a matrix describing the imaging device, $\|\cdot\|_{T V}$ is the total variation norm, $\|\cdot\|_{1}$ is the $\ell_{1}$ norm, $\Psi$ is a wavelet transform, and $\alpha>0$ and $\beta>0$ are weights. The first term in the objective is the data fidelity term, while the next two terms are for regularization; they are designed to enhance edges and increase image sparsity. In our experiments, $\boldsymbol{\Psi}$ is a normalized Haar wavelet with four levels and $\boldsymbol{\Psi} \boldsymbol{\Psi}^{\top}=I$. The problem (6.1) is equivalent to

$$
\min _{(\mathbf{u}, \mathbf{v}, \mathbf{w})} \frac{1}{2}\|\mathbf{F u}-\mathbf{f}\|^{2}+\alpha\|\mathbf{w}\|_{1,2}+\beta\|\mathbf{v}\|_{1} \text { subject to } \mathbf{B u}=\mathbf{w}, \Psi^{\top} \mathbf{u}=\mathbf{v},
$$

where $\mathbf{B u}=\nabla \mathbf{u}$ and $(\nabla \mathbf{u})_{i}$ is the vector of finite differences in the image along the coordinate directions at the i-th pixel in the image, $\|\mathbf{w}\|_{1,2}=\sum_{i=1}^{N}\left\|(\nabla \mathbf{u})_{i}\right\|_{2}$, and $N$ is the total number of pixels in the image.

The problem (6.2) has the structure appearing in (1.1) -(1.2) with $h_{1}:=0, f_{1}(\mathbf{u})=$ $1 / 2\|\mathbf{F u}-\mathbf{f}\|^{2}, h_{2}(\mathbf{w})=\|\mathbf{w}\|_{1,2}, f_{2}:=0, h_{3}(\mathbf{v})=\|\mathbf{v}\|_{1}, f_{3}:=0$,

$$
\mathbf{A}_{1}=\left(\begin{array}{l}
\mathbf{B} \\
\mathbf{\Psi}^{\top}
\end{array}\right), \quad \mathbf{A}_{2}=\left(\begin{array}{r}
-\mathbf{I} \\
\mathbf{0}
\end{array}\right), \quad \mathbf{A}_{3}=\left(\begin{array}{r}
\mathbf{0} \\
-\mathbf{I}
\end{array}\right), \quad \text { and } \quad \mathbf{b}=\left(\begin{array}{l}
\mathbf{0} \\
\mathbf{0}
\end{array}\right) .
$$

The algorithm parameters $\alpha^{l}$ and $\delta^{l}$ were chosen as in (2.5). Since $f_{2}=f_{3}=0$, the second and third subproblems are solved in closed form, due to the simple structure of $h_{2}$ and $h_{3}$. Only the first subproblem is solved inexactly. At iteration $k$, the solution of this subproblem approximates the solution of

$$
\min _{\mathbf{u}} \frac{1}{2}\|\mathbf{F u}-\mathbf{f}\|^{2}+\frac{\rho}{2}\left\|\mathbf{B u}-\mathbf{w}^{k}+\rho^{-1} \boldsymbol{\lambda}^{k}\right\|^{2}+\frac{\rho}{2}\left\|\Psi^{\top} \mathbf{u}-\mathbf{v}^{k}+\rho^{-1} \boldsymbol{\mu}^{k}\right\|^{2},
$$

where $\boldsymbol{\lambda}^{k}$ and $\boldsymbol{\mu}^{k}$ are the Lagrange multipliers at iteration $k$ for the constraints $\mathbf{B u}=$ $\mathbf{w}$ and $\boldsymbol{\Psi}^{\top} \mathbf{u}=\mathbf{v}$ respectively. Details of the experimental setup can be found in [21]. 
(a)

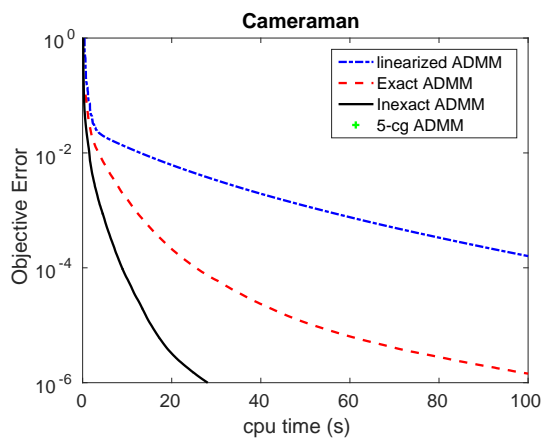

(c)

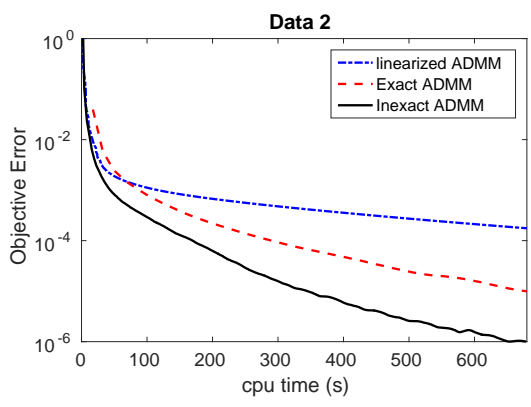

(b)

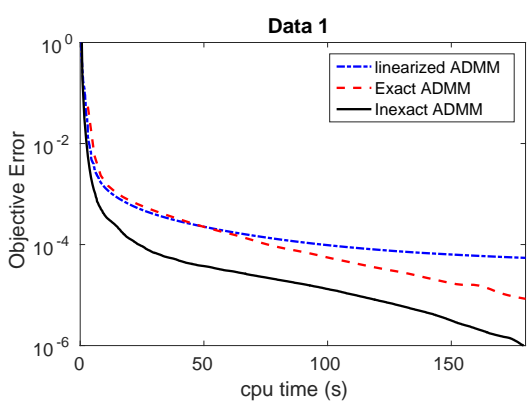

(d)

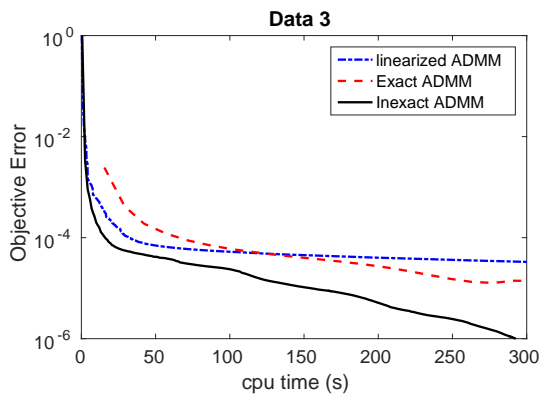

FIG. 6.1. Base-10 logarithm of the relative objective error versus CPU time for the test problems.

The $i$-th block diagonal element of $\mathbf{Q}$ was taken to be a multiple $\gamma_{i}$ of the identity I. According to the assumptions of IADM, $\gamma_{1}$ should be chosen large enough that $\gamma_{1} \mathbf{I}-\mathbf{A}_{1}^{\top} \mathbf{A}_{1}$ is positive semidefinite, where

$$
\mathbf{A}_{1}^{\top} \mathbf{A}_{1}=\mathbf{B}^{\top} \mathbf{B}+\Psi \Psi^{\top} .
$$

However, a closer inspection of the global convergence proof reveals that for convergence, it is sufficient to have

$$
\gamma_{1}\left\|\mathbf{z}^{k}-\mathbf{y}^{k}\right\|^{2} \geq\left\|\mathbf{A}_{1}\left(\mathbf{z}^{k}-\mathbf{y}^{k}\right)\right\|^{2}
$$

in each iteration. Instead of computing the largest eigenvalue of $\mathbf{A}_{1}^{\top} \mathbf{A}_{1}$, we simply start with $\gamma_{1}=4$ and multiply it by a constant factor (3 in the experiments) whenever the inequality (6.3) is violated. Within a finite number of iterations, $\gamma_{1}$ is large enough that (6.3) always holds.

Figure 6.1plots the logarithm of the relative objective error versus the CPU time for the four test problems and the three methods. Note that the first few iterations of the exact ADMM for Data 3 have error greater than one, so they missing from the plot. Observe that I-ADMM performed better than the exact ADMM and the exact ADMM was generally better than the single linearization step, except possibly in the initial iterations where the high accuracy of the exact ADMM was not helpful. I-ADMM gave better performance both initially and asymptotically.

7. Conclusion. We propose an inexact alternating direction method of multipliers, I-ADMM, for solving separable convex linearly constrained optimization problems, where the objective is the sum of smooth and relatively simple nonsmooth 
terms. The nonsmooth terms could be infinite, so the algorithms and analysis include problems with additional convex constraints. This I-ADMM emanates for our earlier work [10, 20, 21] on a Bregman Operator Splitting algorithm with a variable stepsize (BOSVS). The subproblems are solved using an accelerated gradient algorithm that employs a linearization of both the smooth objective and the penalty term. We establish an $\mathcal{O}(1 / k)$ ergodic convergence rate for I-ADMM, where $k$ is the iteration number. Under a strong convexity assumption, the convergence rate improves to $\mathcal{O}\left(1 / k^{2}\right)$ for both ergodic and nonergodic iterates. When an error bound condition holds, 2 -step linear convergence is established for nonergodic iterates. The convergence rates for I-ADMM are consistent with convergence rates obtained for exact ADMM schemes such as those in [23, 28, 30, 35, 38, 42. As observed in the numerical experiments, an advantage of the inexact scheme is that the computing time to achieve a given error tolerance is reduced, when compared to the the exact iteration, since the accuracy of the subproblem solutions are adaptively increased as the iterates converge so as to achieve the same convergence rates as the exact algorithms.

8. Appendix: Proofs for the Global Convergence Analysis. For reference, given a smooth function $\Psi: \mathbb{R}^{n} \rightarrow \mathbb{R}$ and a convex real-valued function $h$ with convexity modulus $\mu$, the first-order optimality condition for a minimizer $\mathbf{u}$ of the $\operatorname{sum} \Psi(\cdot)+h(\cdot)$ is given by

$$
h(\mathbf{u})+(\mu / 2)\|\mathbf{w}-\mathbf{u}\|^{2} \leq h(\mathbf{w})+\nabla \Psi(\mathbf{u})(\mathbf{w}-\mathbf{u})
$$

for every $\mathbf{w} \in \mathbb{R}^{n}$.

Proof of Lemma 3.1, By the definition $\mathbf{a}_{i}^{l}=\left(1-\alpha^{l}\right) \mathbf{a}_{i}^{l-1}+\alpha^{l} \mathbf{u}_{i}^{l}$, we have

$$
\left\langle\nabla f_{i}\left(\overline{\mathbf{a}}_{i}^{l}\right), \mathbf{a}_{i}^{l}-\overline{\mathbf{a}}_{i}^{l}\right\rangle=\left(1-\alpha^{l}\right)\left\langle\nabla f_{i}\left(\overline{\mathbf{a}}_{i}^{l}\right), \mathbf{a}_{i}^{l-1}-\overline{\mathbf{a}}_{i}^{l}\right\rangle+\alpha^{l}\left\langle\nabla f_{i}\left(\overline{\mathbf{a}}_{i}^{l}\right), \mathbf{u}_{i}^{l}-\overline{\mathbf{a}}_{i}^{l}\right\rangle .
$$

Add to this the identity $f_{i}\left(\overline{\mathbf{a}}_{i}^{l}\right)=\left(1-\alpha^{l}\right) f_{i}\left(\overline{\mathbf{a}}_{i}^{l}\right)+\alpha^{l} f_{i}\left(\overline{\mathbf{a}}_{i}^{l}\right)$ to obtain

$$
\begin{gathered}
f_{i}\left(\overline{\mathbf{a}}_{i}^{l}\right)+\left\langle\nabla f_{i}\left(\overline{\mathbf{a}}_{i}^{l}\right), \mathbf{a}_{i}^{l}-\overline{\mathbf{a}}_{i}^{l}\right\rangle= \\
\left(1-\alpha^{l}\right)\left[f_{i}\left(\overline{\mathbf{a}}_{i}^{l}\right)+\left\langle\nabla f_{i}\left(\overline{\mathbf{a}}_{i}^{l}\right), \mathbf{a}_{i}^{l-1}-\overline{\mathbf{a}}_{i}^{l}\right\rangle\right]+\alpha^{l}\left[f_{i}\left(\overline{\mathbf{a}}_{i}^{l}\right)+\left\langle\nabla f_{i}\left(\overline{\mathbf{a}}_{i}^{l}\right), \mathbf{u}_{i}^{l}-\overline{\mathbf{a}}_{i}^{l}\right\rangle\right] .
\end{gathered}
$$

By the convexity of $f_{i}$, it follows that $f_{i}\left(\overline{\mathbf{a}}_{i}^{l}\right)+\left\langle\nabla f_{i}\left(\overline{\mathbf{a}}_{i}^{l}\right), \mathbf{a}_{i}^{l-1}-\overline{\mathbf{a}}_{i}^{l}\right\rangle \leq f_{i}\left(\mathbf{a}_{i}^{l-1}\right)$. Hence,

$$
f_{i}\left(\overline{\mathbf{a}}_{i}^{l}\right)+\left\langle\nabla f_{i}\left(\overline{\mathbf{a}}_{i}^{l}\right), \mathbf{a}_{i}^{l}-\overline{\mathbf{a}}_{i}^{l}\right\rangle \leq\left(1-\alpha^{l}\right) f_{i}\left(\mathbf{a}_{i}^{l-1}\right)+\alpha^{l}\left[f_{i}\left(\overline{\mathbf{a}}_{i}^{l}\right)+\left\langle\nabla f_{i}\left(\overline{\mathbf{a}}_{i}^{l}\right), \mathbf{u}_{i}^{l}-\overline{\mathbf{a}}_{i}^{l}\right\rangle\right] .
$$

Adding and subtracting any $\mathbf{u} \in \mathbb{R}^{n_{i}}$ in the last term, and then exploiting the convexity of $f_{i}$ gives

$$
\begin{aligned}
f_{i}\left(\overline{\mathbf{a}}_{i}^{l}\right)+\left\langle\nabla f_{i}\left(\overline{\mathbf{a}}_{i}^{l}\right), \mathbf{u}_{i}^{l}-\overline{\mathbf{a}}_{i}^{l}\right\rangle & =\left[f_{i}\left(\overline{\mathbf{a}}_{i}^{l}\right)+\left\langle\nabla f_{i}\left(\overline{\mathbf{a}}_{i}^{l}\right), \mathbf{u}-\overline{\mathbf{a}}_{i}^{l}\right\rangle\right]+\left\langle\nabla f_{i}\left(\overline{\mathbf{a}}_{i}^{l}\right), \mathbf{u}_{i}^{l}-\mathbf{u}\right\rangle \\
& \leq f_{i}(\mathbf{u})+\left\langle\nabla f_{i}\left(\overline{\mathbf{a}}_{i}^{l}\right), \mathbf{u}_{i}^{l}-\mathbf{u}\right\rangle .
\end{aligned}
$$

Therefore,

$$
f_{i}\left(\overline{\mathbf{a}}_{i}^{l}\right)+\left\langle\nabla f_{i}\left(\overline{\mathbf{a}}_{i}^{l}\right), \mathbf{a}_{i}^{l}-\overline{\mathbf{a}}_{i}^{l}\right\rangle \leq\left(1-\alpha^{l}\right) f_{i}\left(\mathbf{a}_{i}^{l-1}\right)+\alpha^{l}\left[f_{i}(\mathbf{u})+\left\langle\nabla f_{i}\left(\overline{\mathbf{a}}_{i}^{l}\right), \mathbf{u}_{i}^{l}-\mathbf{u}\right\rangle\right] .
$$

Now by the line search condition in Step 1a of Algorithm 2.2 and then by (8.2), we have

$$
L_{i}^{k}\left(\mathbf{a}_{i}^{l}\right)=f_{i}\left(\mathbf{a}_{i}^{l}\right)+\frac{\rho}{2}\left\|\mathbf{A}_{i} \mathbf{a}_{i}^{l}-\mathbf{b}_{i}^{k}+\lambda^{k} / \rho\right\|^{2}+h_{i}\left(\mathbf{a}_{i}^{l}\right)
$$




$$
\begin{aligned}
& \leq f_{i}\left(\overline{\mathbf{a}}_{i}^{l}\right)+\left\langle\nabla f_{i}\left(\overline{\mathbf{a}}_{i}^{l}\right), \mathbf{a}_{i}^{l}-\overline{\mathbf{a}}_{i}^{l}\right\rangle+\frac{(1-\sigma) \delta^{l}}{2 \alpha^{l}}\left\|\mathbf{a}_{i}^{l}-\overline{\mathbf{a}}_{i}^{l}\right\|^{2} \\
&+\frac{\rho}{2}\left\|\mathbf{A}_{i} \mathbf{a}_{i}^{l}-\mathbf{b}_{i}^{k}+\boldsymbol{\lambda}^{k} / \rho\right\|^{2}+h_{i}\left(\mathbf{a}_{i}^{l}\right) \\
& \leq\left(1-\alpha^{l}\right) f_{i}\left(\mathbf{a}_{i}^{l-1}\right)+\alpha^{l} f_{i}(\mathbf{u})+\alpha^{l}\left\langle\nabla f_{i}\left(\overline{\mathbf{a}}_{i}^{l}\right), \mathbf{u}_{i}^{l}-\mathbf{u}\right\rangle+\frac{(1-\sigma) \delta^{l}}{2 \alpha^{l}}\left\|\mathbf{a}_{i}^{l}-\overline{\mathbf{a}}_{i}^{l}\right\|^{2} \\
& \quad+\frac{\rho}{2}\left\|\mathbf{A}_{i} \mathbf{a}_{i}^{l}-\mathbf{b}_{i}^{k}+\boldsymbol{\lambda}^{k} / \rho\right\|^{2}+h_{i}\left(\mathbf{a}_{i}^{l}\right) .
\end{aligned}
$$

Next, we utilize the definitions of $\mathbf{a}_{i}^{l}$ and $\overline{\mathbf{a}}_{i}^{l}$, and the convexity of both $h_{i}$ and the norm term to obtain

$$
\begin{aligned}
& L_{i}^{k}\left(\mathbf{a}_{i}^{l}\right) \leq(1\left.-\alpha^{l}\right) f_{i}\left(\mathbf{a}_{i}^{l-1}\right)+\alpha^{l}\left[f_{i}(\mathbf{u})+\left\langle\nabla f_{i}\left(\overline{\mathbf{a}}_{i}^{l}\right), \mathbf{u}_{i}^{l}-\mathbf{u}\right\rangle\right]+\frac{(1-\sigma) \delta^{l}}{2 \alpha^{l}}\left\|\mathbf{a}_{i}^{l}-\overline{\mathbf{a}}_{i}^{l}\right\|^{2} \\
&+\left(1-\alpha^{l}\right)\left(\frac{\rho}{2}\left\|\mathbf{A}_{i} \mathbf{a}_{i}^{l-1}-\mathbf{b}_{i}^{k}+\boldsymbol{\lambda}^{k} / \rho\right\|^{2}+h_{i}\left(\mathbf{a}_{i}^{l-1}\right)\right) \\
&+\alpha^{l}\left(\frac{\rho}{2}\left\|\mathbf{A}_{i} \mathbf{u}_{i}^{l}-\mathbf{b}_{i}^{k}+\boldsymbol{\lambda}^{k} / \rho\right\|^{2}+h_{i}\left(\mathbf{u}_{i}^{l}\right)\right) \\
&=\left(1-\alpha^{l}\right)\left(f_{i}\left(\mathbf{a}_{i}^{l-1}\right)+\frac{\rho}{2}\left\|\mathbf{A}_{i} \mathbf{a}_{i}^{l-1}-\mathbf{b}_{i}^{k}+\boldsymbol{\lambda}^{k} / \rho\right\|^{2}+h_{i}\left(\mathbf{a}_{i}^{l-1}\right)\right) \\
&+\alpha^{l}\left[f_{i}(\mathbf{u})+\left\langle\nabla f_{i}\left(\overline{\mathbf{a}}_{i}^{l}\right), \mathbf{u}_{i}^{l}-\mathbf{u}\right\rangle\right]+\frac{(1-\sigma) \delta^{l} \alpha^{l}}{2}\left\|\mathbf{u}_{i}^{l}-\mathbf{u}_{i}^{l-1}\right\|^{2} \\
&+\alpha^{l}\left(\frac{\rho}{2}\left\|\mathbf{A}_{i} \mathbf{u}_{i}^{l}-\mathbf{b}_{i}^{k}+\boldsymbol{\lambda}^{k} / \rho\right\|^{2}+h_{i}\left(\mathbf{u}_{i}^{l}\right)\right) \\
&=\left(1-\alpha^{l}\right) L_{i}^{k}\left(\mathbf{a}_{i}^{l-1}\right)+\alpha^{l}\left[f_{i}(\mathbf{u})+\left\langle\nabla f_{i}\left(\overline{\mathbf{a}}_{i}^{l}\right), \mathbf{u}_{i}^{l}-\mathbf{u}\right\rangle\right] \\
& \quad+\frac{(1-\sigma) \delta^{l} \alpha^{l}}{2}\left\|\mathbf{u}_{i}^{l}-\mathbf{u}_{i}^{l-1}\right\|^{2}+\alpha^{l}\left(\frac{\rho}{2}\left\|\mathbf{A}_{i} \mathbf{u}_{i}^{l}-\mathbf{b}_{i}^{k}+\boldsymbol{\lambda}^{k} / \rho\right\|^{2}+h_{i}\left(\mathbf{u}_{i}^{l}\right)\right) .
\end{aligned}
$$

By (8.1), the first-order optimality condition for $\mathbf{u}_{i}^{l}$ in Step 1a is

$$
\begin{aligned}
& h_{i}\left(\mathbf{u}_{i}^{l}\right)+\left(\mu_{i} / 2\right)\left\|\mathbf{u}-\mathbf{u}_{i}^{l}\right\|^{2} \\
\leq & \left\langle\nabla P\left(\mathbf{u}_{i}^{l}\right),\left(\mathbf{u}-\mathbf{u}_{i}^{l}\right)\right\rangle+\rho\left\langle\mathbf{u}_{i}^{l}-\mathbf{y}_{i}^{k}, \overline{\mathbf{Q}}_{i}\left(\mathbf{u}-\mathbf{u}_{i}^{l}\right)\right\rangle+h_{i}(\mathbf{u}),
\end{aligned}
$$

where

$$
\nabla P\left(\mathbf{u}_{i}^{l}\right)=\nabla f_{i}\left(\overline{\mathbf{a}}_{i}^{l}\right)+\delta^{l}\left(\mathbf{u}_{i}^{l}-\mathbf{u}_{i}^{l-1}\right)+\rho\left(\mathbf{A}_{i}^{\top}\left(\mathbf{A}_{i} \mathbf{u}_{i}^{l}-\mathbf{b}_{i}^{k}+\boldsymbol{\lambda}^{k} / \rho\right) .\right.
$$

Multiply (8.4) by $\alpha^{l}$ and add to (8.3) to obtain (after some algebra):

$$
\begin{gathered}
L_{i}^{k}\left(\mathbf{a}_{i}^{l}\right)+\left(\alpha^{l} \mu_{i} / 2\right)\left\|\mathbf{u}-\mathbf{u}_{i}^{l}\right\|^{2} \leq \\
\left(1-\alpha^{l}\right) L_{i}^{k}\left(\mathbf{a}_{i}^{l-1}\right)+\alpha^{l} L_{i}^{k}(\mathbf{u})+\frac{\delta^{l} \alpha^{l}}{2}\left(\left\|\mathbf{u}-\mathbf{u}_{i}^{l-1}\right\|^{2}-\left\|\mathbf{u}-\mathbf{u}_{i}^{l}\right\|^{2}\right) \\
-\frac{\sigma \delta^{l} \alpha^{l}}{2}\left\|\mathbf{u}_{i}^{l}-\mathbf{u}_{i}^{l-1}\right\|^{2}-\frac{\alpha^{l} \rho}{2}\left\|\mathbf{A}_{i}\left(\mathbf{u}-\mathbf{u}_{i}^{l}\right)\right\|^{2}+\rho \alpha^{l}\left(\mathbf{u}^{l}-\mathbf{y}_{i}^{k}\right)^{\top} \overline{\mathbf{Q}}_{i}\left(\mathbf{u}-\mathbf{u}_{i}^{l}\right) .
\end{gathered}
$$

Hence, for any $\mathbf{u} \in \mathbb{R}^{n_{i}}$ we have

$$
\begin{gathered}
L_{i}^{k}\left(\mathbf{a}_{i}^{l}\right)-L_{i}^{k}(\mathbf{u})+\left(\alpha^{l} \mu_{i} / 2\right)\left\|\mathbf{u}-\mathbf{u}_{i}^{l}\right\|^{2} \leq \\
\left(1-\alpha^{l}\right)\left(L_{i}^{k}\left(\mathbf{a}_{i}^{l-1}\right)-L_{i}^{k}(\mathbf{u})\right)+\frac{\delta^{l} \alpha^{l}}{2}\left(\left\|\mathbf{u}-\mathbf{u}_{i}^{l-1}\right\|^{2}-\left\|\mathbf{u}-\mathbf{u}_{i}^{l}\right\|^{2}\right) \\
-\frac{\sigma \delta^{l} \alpha^{l}}{2}\left\|\mathbf{u}_{i}^{l}-\mathbf{u}_{i}^{l-1}\right\|^{2}-\frac{\alpha^{l} \rho}{2}\left\|\mathbf{A}_{i}\left(\mathbf{u}-\mathbf{u}_{i}^{l}\right)\right\|^{2}+\rho \alpha^{l}\left(\mathbf{u}^{l}-\mathbf{y}_{i}^{k}\right)^{\top} \overline{\mathbf{Q}}_{i}\left(\mathbf{u}-\mathbf{u}^{l}\right) .
\end{gathered}
$$


From the definition of $\gamma^{l}$ in Algorithm 2.2, it follows that $\left(1-\alpha^{l}\right) \gamma^{l}=\gamma^{l-1}$ with the convention that $\gamma^{0}=0$ (since $\alpha^{1}=1$ ). Hence, for any sequence $d^{l}, l \geq 0$, we have

$$
\sum_{l=1}^{j}\left(\gamma^{l} d^{l}-\left(1-\alpha^{l}\right) \gamma^{l} d^{l-1}\right)=\sum_{l=1}^{j}\left(\gamma^{l} d^{l}-\gamma^{l-1} d^{l-1}\right)=\gamma^{j} d^{j}
$$

Suppose that $d^{l} \geq 0$ for each $l$. By assumption, $\xi^{l}=\gamma^{l} \delta^{l} \alpha^{l}$ is nonincreasing; since $\alpha^{1}=1$ and $\gamma^{1}=1 / \delta^{1}$, it follows that $\xi^{1}=1$, and we have

$$
\begin{aligned}
\sum_{l=1}^{j} \xi^{l}\left(d^{l}-d^{l-1}\right) & =d^{1}-d^{0}+\sum_{l=2}^{j} \xi^{l}\left(d^{l}-d^{l-1}\right) \\
& \geq d^{1}-d^{0}+\sum_{l=2}^{j}\left(\xi^{l} d^{l}-\xi^{l-1} d^{l-1}\right)=\xi^{j} d^{j}-d^{0} .
\end{aligned}
$$

We now multiply (8.5) by $\gamma^{l}$ and sum over $l$ between 1 and $L$. Exploiting the identity (8.6) with $d^{l}=L_{i}^{k}\left(\mathbf{a}_{i}^{l}\right)-L_{i}^{k}(\mathbf{u})$ and (8.7) with $d^{l}=\left\|\mathbf{u}_{i}^{l}-\mathbf{u}\right\|^{2}$, we obtain

$$
\begin{gathered}
L_{i}^{k}(\mathbf{u})-L_{i}^{k}\left(\mathbf{a}_{i}^{L}\right) \geq \frac{1}{2 \gamma^{L}}\left(\xi^{L}\left\|\mathbf{u}-\mathbf{u}_{i}^{L}\right\|^{2}-\left\|\mathbf{u}-\mathbf{u}_{i}^{0}\right\|^{2}\right) \\
+\frac{\sigma}{2 \gamma^{L}} \sum_{l=1}^{L} \xi^{l}\left\|\mathbf{u}_{i}^{l}-\mathbf{u}_{i}^{l-1}\right\|^{2}+\frac{\rho}{2 \gamma^{L}} \sum_{l=1}^{L}\left(\gamma^{l} \alpha^{l}\right)\left\|\mathbf{A}_{i}\left(\mathbf{u}-\mathbf{u}_{i}^{l}\right)\right\|^{2} \\
+\frac{\rho}{\gamma^{L}} \sum_{l=1}^{L}\left(\gamma^{l} \alpha^{l}\right)\left(\mathbf{u}_{i}^{l}-\mathbf{y}_{i}^{k}\right)^{\top} \overline{\mathbf{Q}}_{i}\left(\mathbf{u}_{i}^{l}-\mathbf{u}\right)+\frac{\mu_{i}}{2 \gamma^{L}} \sum_{l=1}^{L}\left(\gamma^{l} \alpha^{l}\right)\left\|\mathbf{u}-\mathbf{u}_{i}^{l}\right\|^{2} .
\end{gathered}
$$

Next, we multiply the definition $\mathbf{a}_{i}^{j}=\left(1-\alpha^{j}\right) \mathbf{a}_{i}^{j-1}+\alpha^{j} \mathbf{u}_{i}^{j}$ by $\gamma^{j}$ and sum over $j$ between 1 and $l$. Again, exploiting the identity $\left(1-\alpha^{j}\right) \gamma^{j}=\gamma^{j-1}$ yields

$$
\mathbf{a}_{i}^{l}=\frac{1}{\gamma^{l}} \sum_{j=1}^{l}\left(\gamma^{j} \alpha^{j}\right) \mathbf{u}_{i}^{j}
$$

Since $\alpha^{j} \gamma^{j}=\gamma^{j}-\gamma^{j-1}$, it follows that

$$
\gamma^{l}=\sum_{j=1}^{l} \alpha^{j} \gamma^{j}
$$

Consequently, $\mathbf{a}_{i}^{l}$ is a convex combination of $\mathbf{u}_{i}^{1}$ through $\mathbf{u}_{i}^{l}$. Since $\left\|\mathbf{A}_{i}(\mathbf{u}-\mathbf{w})\right\|^{2}$, $\left(\mathbf{w}-\mathbf{y}_{i}^{k}\right)^{\top} \overline{\mathbf{Q}}_{i}(\mathbf{w}-\mathbf{u})$, and $\|\mathbf{u}-\mathbf{w}\|^{2}$ are convex functions of $\mathbf{w}$, Jensen's inequality can be applied to each of the last three terms in (8.8). For example, we have

$$
\frac{1}{\gamma^{L}} \sum_{l=1}^{L}\left(\gamma^{l} \alpha^{l}\right)\left\|\mathbf{u}-\mathbf{u}_{i}^{l}\right\|^{2} \geq\left\|\mathbf{u}-\mathbf{a}_{i}^{L}\right\|^{2} .
$$

The net effect of Jensen's inequality is to delete the summation and replace $\mathbf{u}_{i}^{l}$ by $\mathbf{a}_{i}^{L}$ in the last three terms of (8.8) to obtain

$$
\begin{gathered}
L_{i}^{k}(\mathbf{u})-L_{i}^{k}\left(\mathbf{a}_{i}^{L}\right) \geq \frac{1}{2 \gamma^{L}}\left(\xi^{L}\left\|\mathbf{u}-\mathbf{u}_{i}^{L}\right\|^{2}-\left\|\mathbf{u}-\mathbf{u}_{i}^{0}\right\|^{2}\right) \\
+\frac{\rho}{2}\left\|\mathbf{A}_{i}\left(\mathbf{u}-\mathbf{a}_{i}^{L}\right)\right\|^{2}+\rho\left(\mathbf{a}_{i}^{L}-\mathbf{y}_{i}^{k}\right)^{\top} \overline{\mathbf{Q}}_{i}\left(\mathbf{a}_{i}^{L}-\mathbf{u}\right)+\frac{\mu_{i}}{2}\left\|\mathbf{u}-\mathbf{a}_{i}^{L}\right\|^{2}+\frac{\sigma}{2 \gamma^{L}} \sum_{l=1}^{L} \xi^{l}\left\|\mathbf{u}_{i}^{l}-\mathbf{u}_{i}^{l-1}\right\|^{2} .
\end{gathered}
$$


Hence, after discarding the $\mathbf{u}_{i}^{L}$ term, we have

$$
\begin{aligned}
\bar{L}_{i}^{k}(\mathbf{u})-\bar{L}_{i}^{k}\left(\mathbf{a}_{i}^{L}\right) \geq & \frac{-1}{2 \gamma^{L}}\left\|\mathbf{u}-\mathbf{u}_{i}^{0}\right\|^{2}+\frac{\sigma}{2 \gamma^{L}} \sum_{l=1}^{L} \xi^{l}\left\|\mathbf{u}_{i}^{l}-\mathbf{u}_{i}^{l-1}\right\|^{2} \\
& +\frac{\rho}{2}\left\|\mathbf{A}_{i}\left(\mathbf{u}-\mathbf{a}_{i}^{L}\right)\right\|^{2}+\rho\left(\mathbf{a}_{i}^{L}-\mathbf{y}_{i}^{k}\right)^{\top} \overline{\mathbf{Q}}_{i}\left(\mathbf{a}_{i}^{L}-\mathbf{u}\right) \\
& +\frac{\rho}{2}\left\|\mathbf{u}-\mathbf{y}_{i}^{k}\right\|_{\mathbf{Q}_{i}}^{2}-\frac{\rho}{2}\left\|\mathbf{a}_{i}^{L}-\mathbf{y}_{i}^{k}\right\|_{\mathbf{Q}_{i}}^{2}+\frac{\mu_{i}}{2}\left\|\mathbf{u}-\mathbf{a}_{i}^{L}\right\|^{2} \\
= & \frac{-1}{2 \gamma^{L}}\left\|\mathbf{u}-\mathbf{u}_{i}^{0}\right\|^{2}+\frac{\sigma}{2 \gamma^{L}} \sum_{l=1}^{L} \xi^{l}\left\|\mathbf{u}_{i}^{l}-\mathbf{u}_{i}^{l-1}\right\|^{2} \\
& +\frac{\rho}{2}\left\|\mathbf{A}_{i}\left(\mathbf{u}-\mathbf{a}_{i}^{L}\right)\right\|^{2}+\frac{\rho}{2}\left\|\mathbf{u}-\mathbf{a}_{i}^{L}\right\|_{\overline{\mathbf{Q}}_{i}}^{2}+\frac{\mu_{i}}{2}\left\|\mathbf{u}-\mathbf{a}_{i}^{L}\right\|^{2} \\
= & \frac{-1}{2 \gamma^{L}}\left\|\mathbf{u}-\mathbf{u}_{i}^{0}\right\|^{2}+\frac{\sigma}{2 \gamma^{L}} \sum_{l=1}^{L} \xi^{l}\left\|\mathbf{u}_{i}^{l}-\mathbf{u}_{i}^{l-1}\right\|^{2} \\
& +\frac{\rho}{2}\left\|\mathbf{u}-\mathbf{a}_{i}^{L}\right\|_{\mathbf{Q}_{i}}^{2}+\frac{\mu_{i}}{2}\left\|\mathbf{u}-\mathbf{a}_{i}^{L}\right\|^{2} .
\end{aligned}
$$

Since $\bar{L}_{i}^{k}(\mathbf{u})-\bar{L}_{i}^{k}\left(\mathbf{a}_{i}^{L}\right) \leq 0$ when $\mathbf{u}=\overline{\mathbf{x}}_{i}^{k}$ and since $\mathbf{u}_{i}^{0}=\mathbf{x}_{i}^{k}$, the proof is complete.

Proof of Lemma 3.2. Let us insert in (8.10) $L=l_{i}^{k}$, the terminating value for $l$ in Algorithm 2.2. In addition, substituting $\mathbf{u}=\mathbf{x}_{i}^{*}, \xi^{l}=1, \mathbf{a}_{i}^{L}=\mathbf{z}_{k}^{k}$, and $\mathbf{u}_{i}^{L}=\mathbf{x}_{i}^{k+1}$, we obtain

$$
L_{i}^{k}\left(\mathbf{x}_{i}^{*}\right)-F_{i}^{k}\left(\mathbf{z}_{i}^{k}\right) \geq \frac{1}{2 \Gamma_{i}^{k}}\left(\left\|\mathbf{x}_{e, i}^{k+1}\right\|^{2}-\left\|\mathbf{x}_{e, i}^{k}\right\|^{2}\right)+\frac{\sigma}{2 \Gamma_{i}^{k}} \sum_{l=1}^{l_{i}^{k}}\left\|\mathbf{u}_{i}^{l}-\mathbf{u}_{i}^{l-1}\right\|^{2},
$$

where $\mathbf{x}_{e}^{k}=\mathbf{x}_{i}^{k}-\mathbf{x}_{i}^{*}$ and

$$
F_{i}^{k}\left(\mathbf{z}_{i}^{k}\right)=L_{i}^{k}\left(\mathbf{z}_{i}^{k}\right)+\frac{\rho}{2}\left\|\mathbf{A}_{i}\left(\mathbf{z}_{i}^{k}-\mathbf{x}_{i}^{*}\right)\right\|^{2}+\rho\left(\mathbf{z}_{i}^{k}-\mathbf{y}_{i}^{k}\right)^{\top} \overline{\mathbf{Q}}_{i}\left(\mathbf{z}_{i}^{k}-\mathbf{x}_{i}^{*}\right)+\frac{\mu_{i}}{2}\left\|\mathbf{z}_{i}^{k}-\mathbf{x}_{i}^{*}\right\|^{2} .
$$

Now, by the definition of $L_{i}^{k}$, a Taylor expansion yields

$$
L_{i}^{k}\left(\mathbf{x}_{i}^{*}\right)-L_{i}^{k}\left(\mathbf{z}_{i}^{k}\right)=
$$

$$
f_{i}\left(\mathbf{x}_{i}^{*}\right)+h_{i}\left(\mathbf{x}_{i}^{*}\right)-f_{i}\left(\mathbf{z}_{i}^{k}\right)-h_{i}\left(\mathbf{z}_{i}^{k}\right)-\rho\left\langle\mathbf{A}_{i} \mathbf{x}_{i}^{*}-\mathbf{b}_{i}^{k}+\lambda^{k} / \rho, \mathbf{A}_{i} \mathbf{z}_{e, i}^{k}\right\rangle-\frac{\rho}{2}\left\|\mathbf{A}_{i} \mathbf{z}_{e, i}^{k}\right\|^{2},
$$

where $\mathbf{z}_{e}^{k}=\mathbf{z}^{k}-\mathbf{x}^{*}$. Observe that

$$
\begin{aligned}
\mathbf{A}_{i} \mathbf{x}_{i}^{*}-\mathbf{b}_{i}^{k} & =-\mathbf{A}_{i} \mathbf{z}_{e, i}^{k}+\mathbf{A}_{i} \mathbf{z}_{i}^{k}-\mathbf{b}+\sum_{j<i} \mathbf{A}_{j} \mathbf{z}_{j}^{k}+\sum_{j>i} \mathbf{A}_{j} \mathbf{y}_{j}^{k} \\
& =-\mathbf{A}_{i} \mathbf{z}_{e, i}^{k}+\sum_{j \leq i} \mathbf{A}_{j} \mathbf{z}_{e, j}^{k}+\sum_{j>i} \mathbf{A}_{j} \mathbf{y}_{e, j}^{k} .
\end{aligned}
$$

where $\mathbf{y}_{e}^{k}=\mathbf{y}^{k}-\mathbf{x}^{*}$. With this substitution in (8.13), we deduce that

$$
\begin{gathered}
L_{i}^{k}\left(\mathbf{x}_{i}^{*}\right)-L_{i}^{k}\left(\mathbf{z}_{i}^{k}\right)-\frac{\rho}{2}\left\|\mathbf{A}_{i} \mathbf{z}_{e, i}^{k}\right\|^{2}= \\
-\Delta_{i}^{k}-\rho\left\langle\sum_{j \leq i} \mathbf{A}_{j} \mathbf{z}_{e, j}^{k}+\sum_{j>i} \mathbf{A}_{j} \mathbf{y}_{e, j}^{k}+\lambda_{e}^{k} / \rho, \mathbf{A}_{i} \mathbf{z}_{e, i}^{k}\right\rangle,
\end{gathered}
$$


where $\boldsymbol{\lambda}_{e}^{k}=\boldsymbol{\lambda}^{k}-\boldsymbol{\lambda}^{*}$, and

$$
\Delta_{i}^{k}=f_{i}\left(\mathbf{z}_{i}^{k}\right)+h_{i}\left(\mathbf{z}_{i}^{k}\right)-f_{i}\left(\mathbf{x}_{i}^{*}\right)-h_{i}\left(\mathbf{x}_{i}^{*}\right)+\left(\boldsymbol{\lambda}^{*}, \mathbf{A}_{i} \mathbf{z}_{e, i}^{k}\right) .
$$

Hence, we have

$$
\begin{aligned}
& L_{i}^{k}\left(\mathbf{x}_{i}^{*}\right)-F_{i}^{k}\left(\mathbf{z}_{i}^{k}\right)=L_{i}^{k}\left(\mathbf{x}_{i}^{*}\right)-L_{i}^{k}\left(\mathbf{z}_{i}^{k}\right)-\frac{\rho}{2}\left\|\mathbf{A}_{i} \mathbf{z}_{e, i}^{k}\right\|^{2}-\rho\left(\mathbf{z}_{i}^{k}-\mathbf{y}_{i}^{k}\right)^{\top} \overline{\mathbf{Q}}_{i} \mathbf{z}_{e, i}^{k}-\frac{\mu_{i}}{2}\left\|\mathbf{z}_{e, i}^{k}\right\|^{2} \\
= & -\Delta_{i}^{k}-\rho\left\langle\sum_{j \leq i} \mathbf{A}_{j} \mathbf{z}_{e, j}^{k}+\sum_{j>i} \mathbf{A}_{j} \mathbf{y}_{e, j}^{k}+\boldsymbol{\lambda}_{e}^{k} / \rho, \mathbf{A}_{i} \mathbf{z}_{e, i}^{k}\right\rangle-\rho\left(\mathbf{z}_{i}^{k}-\mathbf{y}_{i}^{k}\right)^{\top} \overline{\mathbf{Q}}_{i} \mathbf{z}_{e, i}^{k}-\frac{\mu_{i}}{2}\left\|\mathbf{z}_{e, i}^{k}\right\|^{2}
\end{aligned}
$$

where $\Delta_{i}^{k}$ is defined in (8.15). Combining this with the lower bound 8.12) gives

$$
\begin{gathered}
-\rho\left\langle\sum_{j \leq i} \mathbf{A}_{j} \mathbf{z}_{e, j}^{k}+\sum_{j>i} \mathbf{A}_{j} \mathbf{y}_{e, j}^{k}+\lambda_{e}^{k} / \rho, \mathbf{A}_{i} \mathbf{z}_{e, i}^{k}\right\rangle-\Delta_{i}^{k} \geq \\
\frac{1}{2 \Gamma_{i}^{k}}\left(\left\|\mathbf{x}_{e, i}^{k+1}\right\|^{2}-\left\|\mathbf{x}_{e, i}^{k}\right\|^{2}\right)+\frac{\sigma}{2 \Gamma_{i}^{k}} \sum_{l=1}^{l_{i}^{k}}\left\|\mathbf{u}_{i k}^{l}-\mathbf{u}_{i k}^{l-1}\right\|^{2} \\
+\rho\left(\mathbf{z}_{i}^{k}-\mathbf{y}_{i}^{k}\right)^{\top} \overline{\mathbf{Q}}_{i} \mathbf{z}_{e, i}^{k}+\frac{\mu_{i}}{2}\left\|\mathbf{z}_{e, i}^{k}\right\|^{2} .
\end{gathered}
$$

Focusing on the left side of [8.16), observe that

$$
\begin{aligned}
\sum_{j \leq i} \mathbf{A}_{j} \mathbf{z}_{e, j}^{k}+\sum_{j>i} \mathbf{A}_{j} \mathbf{y}_{e, j}^{k} & =\sum_{j=1}^{m} \mathbf{A}_{j}\left(\mathbf{z}_{j}^{k}-\mathbf{x}_{j}^{*}\right)+\sum_{j>i} \mathbf{A}_{j}\left(\mathbf{y}_{j}^{k}-\mathbf{z}_{j}^{k}\right) \\
& =\mathbf{A} \mathbf{z}^{k}-\mathbf{b}+\sum_{j>i} \mathbf{A}_{j}\left(\mathbf{y}_{j}^{k}-\mathbf{z}_{j}^{k}\right)
\end{aligned}
$$

since $\mathbf{A} \mathbf{x}^{*}=\mathbf{b}$. Let $\tau_{i}^{k}$ denote the first part of the right side of (8.16); that is

$$
\tau_{i}^{k}=\frac{1}{2 \Gamma_{i}^{k}}\left(\left\|\mathbf{x}_{e, i}^{k+1}\right\|^{2}-\left\|\mathbf{x}_{e, i}^{k}\right\|^{2}\right)+\frac{\sigma}{2 \Gamma_{i}^{k}} \sum_{l=1}^{l_{i}^{k}}\left\|\mathbf{u}_{i k}^{l}-\mathbf{u}_{i k}^{l-1}\right\|^{2}
$$

With this notation and with the simplification (8.17), (8.16) becomes

$$
\begin{aligned}
& -\rho\left\langle\mathbf{A}_{i} \mathbf{z}_{e, i}^{k}, \mathbf{A} \mathbf{z}^{k}-\mathbf{b}+\boldsymbol{\lambda}_{e}^{k} / \rho+\sum_{j>i} \mathbf{A}_{j}\left(\mathbf{y}_{j}^{k}-\mathbf{z}_{j}^{k}\right)\right\rangle \\
\geq & \tau_{i}^{k}+\Delta_{i}^{k}+\rho\left(\mathbf{z}_{i}^{k}-\mathbf{y}_{i}^{k}\right)^{\top} \overline{\mathbf{Q}}_{i} \mathbf{z}_{e, i}^{k} \\
= & \tau_{i}^{k}+\Delta_{i}^{k}+\rho\left(\mathbf{z}_{i}^{k}-\mathbf{y}_{i}^{k}\right)^{\top}\left(\mathbf{Q}_{i}-\rho \mathbf{A}_{i}^{\top} \mathbf{A}_{i}\right) \mathbf{z}_{e, i}^{k}+\frac{\mu_{i}}{2}\left\|\mathbf{z}_{e, i}^{k}\right\|^{2},
\end{aligned}
$$

which gives

$$
\begin{aligned}
& -\rho\left\langle\mathbf{A}_{i} \mathbf{z}_{e, i}^{k}, \mathbf{A} \mathbf{z}^{k}-\mathbf{b}+\boldsymbol{\lambda}_{e}^{k} / \rho+\sum_{j \geq i} \mathbf{A}_{j}\left(\mathbf{y}_{j}^{k}-\mathbf{z}_{j}^{k}\right)\right\rangle \\
\geq & \tau_{i}^{k}+\Delta_{i}^{k}+\rho\left(\mathbf{z}_{i}^{k}-\mathbf{y}_{i}^{k}\right)^{\top} \mathbf{Q}_{i} \mathbf{z}_{e, i}^{k}+\frac{\mu_{i}}{2}\left\|\mathbf{z}_{e, i}^{k}\right\|^{2} .
\end{aligned}
$$


We will sum the inequality (8.19) over $i$ between 1 and $m$. Let $\mathbf{r}^{k}=\mathbf{A} \mathbf{z}^{k}-\mathbf{b}$, the residual for the linear system. As in (8.17), it follows that

$$
\sum_{i=1}^{m}\left\langle\mathbf{A}_{i} \mathbf{z}_{e, i}^{k}, \mathbf{r}^{k}+\boldsymbol{\lambda}_{e}^{k} / \rho\right\rangle=\left\langle\mathbf{r}^{k}, \mathbf{r}^{k}+\boldsymbol{\lambda}_{e}^{k} / \rho\right\rangle .
$$

Also, observe that

$$
\sum_{j \geq i} \mathbf{A}_{j}\left(\mathbf{y}_{j}^{k}-\mathbf{z}_{j}^{k}\right)=\sum_{j=1}^{m} \mathbf{A}_{j}\left(\mathbf{y}_{j}^{k}-\mathbf{z}_{j}^{k}\right)-\sum_{j=1}^{i-1} \mathbf{A}_{j}\left(\mathbf{y}_{j}^{k}-\mathbf{z}_{j}^{k}\right),
$$

with the convention that the sum from $j=1$ to $j=0$ is 0 . Hence, we have

$$
\begin{aligned}
& \sum_{i=1}^{m}\left(\left\langle\mathbf{A}_{i} \mathbf{z}_{e, i}^{k}, \sum_{j \geq i} \mathbf{A}_{j}\left(\mathbf{y}_{j}^{k}-\mathbf{z}_{j}^{k}\right)\right\rangle-\left(\mathbf{y}_{i}^{k}-\mathbf{z}_{i}^{k}\right)^{\top} \mathbf{Q}_{i} \mathbf{z}_{e, i}^{k}\right) \\
= & \left\langle\mathbf{r}^{k}, \sum_{j=1}^{m} \mathbf{A}_{j} \mathbf{w}_{j}\right\rangle-\sum_{i=1}^{m}\left\langle\mathbf{z}_{e, i}^{k}, \sum_{j=1}^{i-1} \mathbf{A}_{i}^{\top} \mathbf{A}_{j} \mathbf{w}_{j}+\mathbf{Q}_{i} \mathbf{w}_{i}\right\rangle \\
= & \left\langle\mathbf{r}^{k}, \sum_{j=1}^{m} \mathbf{A}_{j} \mathbf{w}_{j}\right\rangle-\left(\mathbf{z}_{e}^{k}\right)^{\top} \mathbf{M} \mathbf{w},
\end{aligned}
$$

where $\mathbf{M}$ is defined in (2.2) and $\mathbf{w}=\mathbf{y}^{k}-\mathbf{z}^{k}$. We sum (8.19) over $i$ between 1 and $m$ and utilize (8.20) and (8.21) to obtain

$$
\begin{gathered}
\left(\mathbf{y}_{e}^{k}\right)^{\top} \mathbf{M} \mathbf{w}-\frac{1}{\rho}\left(\left\langle\mathbf{r}^{k}, \boldsymbol{\lambda}_{e}^{k}\right\rangle+\sum_{i=1}^{m}\left(\tau_{i}^{k}+\Delta_{i}^{k}\right)\right) \\
\geq \mathbf{w}^{\top} \mathbf{M} \mathbf{w}+\left\langle\mathbf{r}^{k}, \mathbf{r}^{k}+\sum_{j=1}^{m} \mathbf{A}_{j} \mathbf{w}_{j}\right\rangle+\sum_{i=1}^{m} \frac{\mu_{i}}{2}\left\|\mathbf{z}_{e, i}^{k}\right\|^{2} .
\end{gathered}
$$

Observe that $\mathbf{M}+\mathbf{M}^{\top}-\mathbf{Q} \succeq \mathbf{A}^{\top} \mathbf{A}$, since $\mathbf{Q}_{i} \succeq \mathbf{A}_{i}^{\top} \mathbf{A}_{i}$ for all $1 \leq i \leq m$. Consequently, we have

$$
\begin{aligned}
\mathbf{w}^{\top} \mathbf{M} \mathbf{w} & =\frac{1}{2} \mathbf{w}^{\top}\left(\mathbf{M}+\mathbf{M}^{\top}\right) \mathbf{w}=\frac{1}{2} \mathbf{w}^{\top}\left(\mathbf{M}+\mathbf{M}^{\top}-\mathbf{Q}\right) \mathbf{w}+\frac{1}{2} \mathbf{w}^{\top} \mathbf{Q} \mathbf{w} \\
& \geq \frac{1}{2}\left\|\sum_{i=1}^{m} \mathbf{A}_{i} \mathbf{w}_{i}\right\|^{2}+\frac{1}{2} \mathbf{w}^{\top} \mathbf{Q} \mathbf{w},
\end{aligned}
$$

which implies that

$$
\mathbf{w}^{\top} \mathbf{M w}+\left\langle\mathbf{r}^{k}, \mathbf{r}^{k}+\sum_{j=1}^{m} \mathbf{A}_{j} \mathbf{w}_{j}\right\rangle \geq \frac{1}{2}\left(\mathbf{w}^{\top} \mathbf{Q} \mathbf{w}+\left\|\mathbf{r}^{k}\right\|^{2}+\left\|\mathbf{r}^{k}+\sum_{i=1}^{m} \mathbf{A}_{i} \mathbf{w}_{i}\right\|^{2}\right) .
$$

Hence, it follows from (8.22) that

$$
\begin{gathered}
\left(\mathbf{y}_{e}^{k}\right)^{\top} \mathbf{M w}-\frac{1}{\rho}\left(\left\langle\mathbf{r}^{k}, \boldsymbol{\lambda}_{e}^{k}\right\rangle+\sum_{i=1}^{m}\left(\tau_{i}^{k}+\Delta_{i}^{k}\right)\right) \\
\geq \frac{1}{2}\left(\|\mathbf{w}\|_{\mathbf{Q}}^{2}+\left\|\mathbf{r}^{k}\right\|^{2}\right)+\sum_{i=1}^{m} \frac{\mu_{i}}{2}\left\|\mathbf{z}_{e, i}^{k}\right\|^{2} .
\end{gathered}
$$


Let $\mathbf{P}=\mathbf{M Q}^{-1} \mathbf{M}^{\top}$ and recall that $\mathbf{w}=\mathbf{y}^{k}-\mathbf{z}^{k}$. By the definition of $\mathbf{y}^{k+1}$ and $\lambda^{k+1}$ in Step 3 of Algorithm 2.1 we have

$$
\begin{gathered}
\left\|\mathbf{y}_{e}^{k}\right\|_{\mathbf{P}}^{2}-\left\|\mathbf{y}_{e}^{k+1}\right\|_{\mathbf{P}}^{2}+\frac{1}{\rho^{2}}\left\|\boldsymbol{\lambda}_{e}^{k}\right\|^{2}-\frac{1}{\rho^{2}}\left\|\boldsymbol{\lambda}_{e}^{k+1}\right\|^{2}= \\
\left\|\mathbf{y}_{e}^{k}\right\|_{\mathbf{P}}^{2}-\left\|\mathbf{y}_{e}^{k}-\alpha \mathbf{M}^{-\top} \mathbf{Q} \mathbf{w}\right\|_{\mathbf{P}}^{2}+\frac{1}{\rho^{2}}\left(\left\|\boldsymbol{\lambda}_{e}^{k}\right\|^{2}-\left\|\boldsymbol{\lambda}_{e}^{k}+\alpha \rho \mathbf{r}^{k}\right\|^{2}\right)= \\
2 \alpha\left(\mathbf{y}_{e}^{k}\right)^{\top} \mathbf{M} \mathbf{w}-\alpha^{2}\|\mathbf{w}\|_{\mathbf{Q}}^{2}-\frac{2 \alpha}{\rho}\left\langle\mathbf{r}^{k}, \boldsymbol{\lambda}_{e}^{k}\right\rangle-\alpha^{2}\left\|\mathbf{r}^{k}\right\|^{2} .
\end{gathered}
$$

On the right side of this equality, we utilize (8.23) multiplied by $2 \alpha$ to conclude that

$$
\begin{gathered}
\left\|\mathbf{y}_{e}^{k}\right\|_{\mathbf{P}}^{2}-\left\|\mathbf{y}_{e}^{k+1}\right\|_{\mathbf{P}}^{2}+\frac{1}{\rho^{2}}\left(\left\|\boldsymbol{\lambda}_{e}^{k}\right\|^{2}-\left\|\boldsymbol{\lambda}_{e}^{k+1}\right\|^{2}\right)-\frac{2 \alpha}{\rho} \sum_{i=1}^{m}\left(\tau_{i}^{k}+\Delta_{i}^{k}\right) \geq \\
\alpha(1-\alpha)\left(\left\|\mathbf{y}^{k}-\mathbf{z}^{k}\right\|_{\mathbf{Q}}^{2}+\left\|\mathbf{r}^{k}\right\|^{2}\right)+\alpha \sum_{i=1}^{m} \mu_{i}\left\|\mathbf{z}_{e, i}^{k}\right\|^{2} .
\end{gathered}
$$

So, by the definition of $\tau_{i}^{k}$, the identity $\Delta^{k}=\sum_{i=1}^{m} \Delta_{i}^{k}$, the inequality (8.24), and the relation $\Gamma_{i}^{k+1} \geq \Gamma_{i}^{k}$ in Steps $1 \mathrm{~b}$ and 1c, it follows that (3.4) holds.

Proof of Theorem 3.3. Since $\xi^{l}=\delta^{l} \alpha^{l} \gamma^{l}=1$ when the parameters $\delta^{l}$ and $\alpha^{l}$ are chosen according to either (2.4) or (2.5), Lemma 3.2 can be utilized. For any $p>0$, we sum the decay property of Lemma 3.2 to obtain

$$
E_{j} \geq E_{j+p}+c \sum_{k=j}^{j+p-1}\left(\left\|\mathbf{y}^{k}-\mathbf{z}^{k}\right\|_{\mathbf{Q}}^{2}+\left\|\mathbf{A} \mathbf{z}^{k}-\mathbf{b}\right\|^{2}+R^{k}\right),
$$

where $c=\alpha \min \{\sigma, \rho(1-\alpha)\}>0$. Let $p$ tend to $+\infty$. Since $\mathbf{Q}$ is positive definite and $R^{k} \geq 0$, it follows from (8.25) that

$$
\lim _{k \rightarrow \infty}\left\|\mathbf{y}^{k}-\mathbf{z}^{k}\right\|=0=\lim _{k \rightarrow \infty}\left\|\mathbf{A} \mathbf{z}^{k}-\mathbf{b}\right\| .
$$

Moreover, by the definition of $E_{k}$ and Lemma 3.2, we know $\mathbf{y}^{k}$ and $\boldsymbol{\lambda}^{k}$ are bounded sequences, and by the first equation in (8.26), $\mathrm{z}^{k}$ is also a bounded sequence. Hence, there exist an infinite sequence $\mathcal{K} \subset\{1,2, \ldots\}$ and limits $\mathbf{x}^{*}$ and $\boldsymbol{\lambda}^{*}$ such that

$$
\lim _{k \in \mathcal{K}} \mathbf{z}^{k}=\mathbf{x}^{*} \quad \text { and } \quad \lim _{k \in \mathcal{K}} \boldsymbol{\lambda}^{k}=\boldsymbol{\lambda}^{*} .
$$

By the first equation in (8.26), we have

$$
\lim _{k \in \mathcal{K}} \mathbf{y}^{k}=\mathbf{x}^{*}
$$

By the second equation in (8.26), $\mathbf{A x}^{*}=\mathbf{b}$. Consequently, by (8.27) and (8.28),

$$
\lim _{k \in \mathcal{K}}\left(\mathbf{A}_{i} \mathbf{z}_{i}^{k}-\mathbf{b}_{i}^{k}\right)=\lim _{k \in \mathcal{K}}\left(\sum_{j \leq i} \mathbf{A}_{j} \mathbf{z}_{j}^{k}+\sum_{j>i} \mathbf{A}_{j} \mathbf{y}_{j}^{k}-\mathbf{b}\right)=\mathbf{A} \mathbf{x}^{*}-\mathbf{b}=\mathbf{0}
$$

for all $i \in[1, m]$. 
The decay property (8.25) also implies that for each $i$,

$$
\lim _{k \rightarrow \infty} r_{i}^{k}=\lim _{k \rightarrow \infty} \frac{1}{\Gamma_{i}^{k}} \sum_{l=1}^{l_{i}^{k}}\left\|\mathbf{u}_{i k}^{l}-\mathbf{u}_{i k}^{l-1}\right\|^{2}=0
$$

where $l_{i}^{k}$ is the terminating value of $l$ in Step $1 \mathrm{~b}$, which exists since the parameters $\delta^{l}$ and $\alpha^{l}$ in Algorithm 2.2 are chosen according to either (2.4) or (2.5). Combine this with (8.26) to conclude that the parameter $\epsilon^{k}$ in Step 2 of Algorithm 2.1 satisfies

$$
\lim _{k \rightarrow \infty} \epsilon^{k}=\lim _{k \rightarrow \infty} \psi\left(\epsilon^{k}\right)=0 .
$$

The remainder of the proof is partitioned into two cases depending on whether the monotone nondecreasing sequence $\Gamma_{i}^{k}$ either approaches a finite limit, or tends to infinity.

Case 1. For some $i, \Gamma_{i}^{k}$ approaches a finite limit. In [21, pp. 227-228] it is shown that $\gamma^{l} \geq l^{2} \Theta$ for some constant $\Theta>0$, independent of $k$. Since $\Gamma_{i}^{k}=\gamma^{l}$ for some $l$, it follows that $l_{i}^{k}$, the terminating value $l$ in Step 1 of Algorithm 2.2, is uniformly bounded when $\Gamma_{i}^{k}$ approaches a finite limit. By (8.30), $\left\|\mathbf{u}_{i k}^{l}-\mathbf{u}_{i k}^{l-1}\right\|$ approaches zero, where the convergence is uniform in $k$ and $l \in\left[1, l_{i}^{k}\right]$. Since $\mathbf{u}_{i k}^{0}=\mathbf{x}_{i}^{k}$, the triangle inequality and the uniform upper bound for $l_{i}^{k}$ imply that $\left\|\mathbf{x}_{i}^{k}-\mathbf{u}_{i k}^{l}\right\|$ approaches zero, where the convergence is uniform in $k$ and $l \in\left[1, l_{i}^{k}\right]$. Since $\mathbf{a}_{i k}^{l}$ is a convex combination of $\mathbf{u}_{i k}^{l}$ (see (8.9) ) for $0 \leq l \leq l_{i}^{k}$ with $l_{i}^{k}$ uniformly bounded and $\left\|\mathbf{x}_{i}^{k}-\mathbf{u}_{i k}^{l}\right\|$ approaching zero, it follows that $\left\|\mathbf{a}_{i k}^{l}-\mathbf{x}_{i}^{k}\right\|$ approaches zero. Since $\mathbf{z}_{i}^{k}=\mathbf{a}_{i k}^{l_{i}^{k}}$ and since $\mathbf{z}_{i}^{k}$ approaches $\mathbf{x}_{i}^{*}$ as $k \in \mathcal{K}$ tends to infinity, we deduce that

$$
\mathbf{x}_{i}^{*}=\lim _{k \in \mathcal{K}} \mathbf{y}_{i}^{k}=\lim _{k \in \mathcal{K}} \mathbf{z}_{i}^{k}=\lim _{k \in \mathcal{K}} \mathbf{x}_{i}^{k}=\lim _{k \in \mathcal{K}} \mathbf{u}_{i k}^{l}=\lim _{k \in \mathcal{K}} \mathbf{a}_{i k}^{l}=\lim _{k \in \mathcal{K}} \overline{\mathbf{a}}_{i k}^{l},
$$

where the last equality is due to the fact that $\overline{\mathbf{a}}_{i k}^{l}$ is a convex combination of $\mathbf{a}_{i k}^{l-1}$ and $\mathbf{u}_{i k}^{l-1}$.

In (8.4) we give the first-order optimality condition for $\mathbf{u}_{i k}^{l}$. Taking the limit as $k \in \mathcal{K}$ tends to infinity and utilizing (8.29) and (8.32), we obtain

$$
h_{i}\left(\mathbf{x}_{i}^{*}\right) \leq h_{i}(\mathbf{u})+\left\langle\nabla f_{i}\left(\mathbf{x}_{i}^{*}\right)+\mathbf{A}_{i}^{\top} \boldsymbol{\lambda}^{*}, \mathbf{u}-\mathbf{x}_{i}^{*}\right\rangle
$$

for every $\mathbf{u} \in \mathbb{R}^{n_{i}}$. Since $\mathbf{A} \mathbf{x}^{*}=\mathbf{b}$ and the first-order optimality conditions are both necessary and sufficient for optimality in this convex setting, it would follow that $\left(\mathbf{x}^{*}, \boldsymbol{\lambda}^{*}\right) \in \mathcal{W}^{*}$ if (8.33) holds for every $i \in[1, m]$. To show that (8.33) holds for all $i$, we need to consider the situation where $\Gamma_{i}^{k}$ tends to infinity.

Case 2. Suppose that $\Gamma_{i}^{k}$ approaches infinity. Let $\overline{\mathbf{x}}_{i}^{k}$ be the minimizer of $\bar{L}_{i}^{k}$ defined in (2.7). Observe that minimizing $\bar{L}_{i}^{k}(\mathbf{u})$ over $\mathbf{u} \in \mathbb{R}^{n_{i}}$ is equivalent to minimizing a sum of the form $g(\mathbf{u})+h_{i}(\mathbf{u})+\left\langle\mathbf{u}, \mathbf{c}^{k}\right\rangle$ where

$$
\mathbf{c}^{k}=\rho \mathbf{A}_{i}^{\top}\left(\mathbf{A}_{i} \mathbf{y}_{i}^{k}-\mathbf{b}_{i}^{k}+\boldsymbol{\lambda}^{k} / \rho\right)-\rho \mathbf{Q}_{i} \mathbf{y}_{i}^{k},
$$

and $g(\mathbf{u})=f_{i}(\mathbf{u})+0.5 \rho\|\mathbf{u}\|_{\mathbf{Q}_{i}}^{2}$. Note that $g$ is smooth and satisfies a strong convexity condition

$$
(\mathbf{u}-\mathbf{v})^{\top}(\nabla g(\mathbf{u})-\nabla g(\mathbf{v})) \geq \rho \nu_{i}\|\mathbf{u}-\mathbf{v}\|^{2}
$$


where $\nu_{i}>0$ is the smallest eigenvalue of $\mathbf{Q}_{i}$. By the strong convexity of $\bar{L}_{i}^{k}$, it has a unique minimizer, and from the first-order optimality conditions and the strong convexity condition (8.34), we obtain the bound

$$
\left\|\overline{\mathbf{x}}_{i}^{j}-\overline{\mathbf{x}}_{i}^{k}\right\| \leq\left\|\mathbf{c}^{j}-\mathbf{c}^{k}\right\| /\left(\rho \nu_{i}\right) .
$$

Since $\mathbf{z}^{k}, \mathbf{y}^{k}$, and $\boldsymbol{\lambda}^{k}$ are bounded sequences, it follows that $\overline{\mathbf{x}}_{i}^{k}$ is a bounded sequence. For $k \in \mathcal{K}$, the sequences $\mathbf{z}^{k}, \mathbf{y}^{k}$, and $\boldsymbol{\lambda}^{k}$ converge to $\mathbf{x}^{*}, \mathbf{x}^{*}$, and $\boldsymbol{\lambda}^{*}$ respectively and $\mathbf{A x}^{*}=\mathbf{b}$, which implies that

$$
\begin{aligned}
\mathbf{c}^{*} & =\lim _{k \in \mathcal{K}} \mathbf{c}^{k}=\mathbf{A}_{i}^{\top}\left[\rho \mathbf{A}_{i} \mathbf{x}_{i}^{*}+\boldsymbol{\lambda}^{*}-\rho\left(\mathbf{b}-\sum_{j \neq i} \mathbf{A}_{j} \mathbf{x}_{j}^{*}\right)\right]-\rho \mathbf{Q}_{i} \mathbf{x}_{i}^{*} \\
& =\mathbf{A}_{i}^{\top} \boldsymbol{\lambda}^{*}-\rho \mathbf{Q}_{i} \mathbf{x}_{i}^{*} .
\end{aligned}
$$

Consequently, by (8.35), $\overline{\mathbf{x}}_{i}^{k}$ for $k \in \mathcal{K}$ forms a Cauchy sequence which approaches a limit.

By (8.31) and the stopping condition in Algorithm (2.2), $\left\|\mathbf{x}_{i}^{k}-\mathbf{z}_{i}^{k}\right\| / \sqrt{\Gamma_{i}^{k}}$ tends to zero as $k$ tends to infinity. By (3.3) and (8.25), $\lambda^{k}$ and $\mathbf{y}^{k}$ are bounded, which implies that $\mathbf{z}^{k}$ is bounded by (8.26). By (8.35), $\overline{\mathbf{x}}_{i}^{k}$ is also bounded. Since $\Gamma_{i}^{k}$ tends to infinity in Case 2 and $\left\|\mathbf{x}_{i}^{k}-\mathbf{z}_{i}^{k}\right\| / \sqrt{\Gamma_{i}^{k}}$ tends to zero, it follows from the triangle inequality and the boundedness of $\overline{\mathbf{x}}_{i}^{k}$ and $\mathbf{z}^{k}$ that $\left\|\mathbf{x}_{i}^{k}-\overline{\mathbf{x}}_{i}^{k}\right\| / \sqrt{\Gamma_{i}^{k}}$ tends to zero as $k$ tends to infinity. Hence, by Lemma 3.1 $\mathbf{z}_{i}^{k}=\mathbf{a}_{i}^{l_{i}^{k}}$ approaches $\overline{\mathbf{x}}_{i}^{k}$ as $k$ tends to infinity; since $\mathbf{z}_{i}^{k}$ approaches $\mathbf{x}_{i}^{*}$ as $k \in \mathcal{K}$ tends to infinity, it follows that $\overline{\mathbf{x}}_{i}^{k}$ approaches $\mathbf{x}_{i}^{*}$ as $k \in \mathcal{K}$ tends to infinity. Let $\overline{\mathbf{x}}_{i}^{*}$ be defined by

$$
\overline{\mathbf{x}}_{i}^{*}=\arg \min _{\mathbf{u}}\left\{g(\mathbf{u})+h_{i}(\mathbf{u})+\left\langle\mathbf{u}, \mathbf{c}^{*}\right\rangle\right\} .
$$

By (8.35) and the fact that $\overline{\mathbf{x}}_{i}^{k}$ approaches $\mathbf{x}_{i}^{*}$ as $k \in \mathcal{K}$ tends to infinity, we conclude that $\overline{\mathbf{x}}_{i}^{*}=\mathbf{x}_{i}^{*}$. In summary, we have

$$
\begin{aligned}
\lim _{k \in \mathcal{K}} \overline{\mathbf{x}}_{i}^{k} & =\mathbf{x}_{i}^{*}=\overline{\mathbf{x}}_{i}^{*}=\arg \min _{\mathbf{u}}\left\{g(\mathbf{u})+h_{i}(\mathbf{u})+\left\langle\mathbf{u}, \mathbf{c}^{*}\right\rangle\right\} . \\
& =\arg \min _{\mathbf{u}}\left\{f_{i}(\mathbf{u})+0.5 \rho\|\mathbf{u}\|_{\mathbf{Q}_{i}}^{2}+h_{i}(\mathbf{u})+\left\langle\mathbf{A}_{i}^{\top} \boldsymbol{\lambda}^{*}-\rho \mathbf{Q}_{i} \mathbf{x}_{i}^{*}, \mathbf{u}\right\rangle\right\} .
\end{aligned}
$$

The first-order optimality conditions for (8.37) are exactly the same as (8.33). This shows that (8.33) holds in Case 1 and Case 2, and $\mathbf{x}^{*}$ is an optimal solution of (1.1)(1.2) with associated multiplier $\boldsymbol{\lambda}^{*}$.

Finally, we need to show that the entire sequence converges. If $\Gamma_{i}^{k}$ is uniformly bounded as in Case 1 , then by (8.32), $\mathbf{x}_{i}^{k}$ approaches $\mathbf{x}_{i}^{*}$ and $\left\|\mathbf{x}_{i}^{k}-\mathbf{x}_{i}^{*}\right\|^{2} / \Gamma_{i}^{k}$ approaches zero as $k$ tends to infinity with $k \in \mathcal{K}$. On the other hand, when $\Gamma_{i}^{k}$ tends to infinity as in Case 2, we showed that $\left\|\mathbf{x}_{i}^{k}-\overline{\mathbf{x}}_{i}^{k}\right\|^{2} / \Gamma_{i}^{k}$ approaches zero and $\overline{\mathbf{x}}_{i}^{k}$ approaches $\mathbf{x}_{i}^{*}$ when $k \in \mathcal{K}$ tends to infinity. Hence, $\left\|\mathbf{x}_{i}^{k}-\mathbf{x}_{i}^{*}\right\|^{2} / \Gamma_{i}^{k}$ approaches zero when $k \in \mathcal{K}$ tends to infinity. Thus in Case 1 and Case $2,\left\|\mathbf{x}_{i}^{k}-\mathbf{x}_{i}^{*}\right\|^{2} / \Gamma_{i}^{k}$ approaches zero as $k \in \mathcal{K}$ tends to infinity. By the definition of $E_{k}$ in (3.4), $E_{k}$ tends to zero as $k \in \mathcal{K}$ tends to infinity. Letting $j$ tend to infinity in (8.25) with $j \in \mathcal{K}$, it follows that $E_{j}$ approaches zero, while the right side of (8.25) shows that the entire sequence $\left(\mathbf{y}^{k}, \boldsymbol{\lambda}^{k}\right)$ approaches $\left(\mathbf{x}^{*}, \boldsymbol{\lambda}^{*}\right)$. By (8.26), the $\mathbf{z}^{k}$ sequence also approaches $\mathbf{x}^{*}$. This completes the proof. 


\section{REFERENCES}

[1] D. P. Bertsekas, Convex Analysis and Optimization, Athena Scientific, Belmont, MA, 2003.

[2] S. Boyd, N. Parikh, E. Chu, B. Peleato, and J. Eckstein, Distributed optimization and statistical learning via the alternating direction method of multipliers, Machine Learning, 3 (2010), pp. 1-122.

[3] X. CAI, D. Han, AND X. YuAn, On the convergence of the direct extension of ADMM for threeblock separable convex minimization models with one strongly convex function, Comput. Optim. Appl., 66 (2017), pp. 39-73.

[4] C. Chen, B. He, Y. Ye, AND X. Yuan, The direct extension of ADMM for multi-block convex minimization problems is not necessarily convergent, Math. Program., 155 (2016), pp. 5779.

[5] C. Chen, M. LI, X. Liu, And Y. Ye, On the convergence of multi-block alternating direction method of multipliers and block coordinate descent method, (2015, arXiv: 1508.00193).

[6] C. Chen, Y. Shen, And Y. You, On the convergence analysis of the alternating direction method of multipliers with three blocks, Abstr. Appl. Anal., 2013 (2013).

[7] G. Chen and M. Teboulle, A proximal-based decomposition method for convex minimization problems, Math. Programming, 64 (1994), pp. 81-101.

[8] J. W. Chen, Y. Y. Wang, H. J. He, And Y. B. Lv, Convergence analysis of positiveindefinite proximal ADMM with a Glowinski's relaxation factor, Numer. Algor., (2019, DOI: $10.1007 / \mathrm{s} 11075-019-00731-9)$.

[9] L. Chen, D. Sun, And K. Toh, An efficient inexact symmetric Gauss-Seidel based majorized ADMM for high-dimensional convex composite conic programming, Math. Program., 161 (2017), pp. 237-270.

[10] Y. Chen, W. W. Hager, M. Yashtini, X. Ye, And H. Zhang, Bregman operator splitting with variable stepsize for total variation image reconstruction, Comput. Optim. Appl., 54 (2013), pp. 317-342.

[11] D. DAVIS AND W. YIN, A three-operator splitting scheme and its optimization applications, Set-Valued and Variational Analysis, 25 (2017), pp. 829-858.

[12] J. Eckstein And D. Bertsekas, On the Douglas-Rachford splitting method and the proximal point algorithm for maximal monotone operators, Mathematical Programming, 55 (1992), pp. 293-318.

[13] J. ECKSTEIN AND P. J. S. Silva, A practical relative error criterion for augmented Lagrangians, Math. Program., 141 (2013), pp. 319-348.

[14] J. ECKSTEIN AND W. YAO, Approximate ADMM algorithms derived from Lagrangian splitting, Comput. Optim. Appl., 68 (2017), pp. 363-405.

[15] - Relative-error approximate versions of DouglasRachford splitting and special cases of the ADMM, Math. Program., 170 (2018), pp. 417-444.

[16] D. GABAY AND B. MerCIER, A dual algorithm for the solution of nonlinear variational problems via finite-element approximations, Comput. Math. Appl., 2 (1976), pp. 17-40.

[17] R. Glowinski, Numerical Methods for Nonlinear Variational Problems, Springer-Verlag, New York, 1984.

[18] D. GoldFARB And S. MA, Fast multiple-splitting algorithms for convex optimization, SIAM J. Optim., 22 (2012), pp. 533-556.

[19] E. Golshtein AND N. TRETYAKov, Modified Lagrangians in convex programming and their generalizations, in Point-to-Set Maps and Mathematical Programming, P. Huard, ed., vol. 10 of Mathematical Programming Studies, Springer Berlin Heidelberg, 1979, pp. 86-97.

[20] W. W. Hager, M. Yashtini, and H. Zhang, An $O(1 / k)$ convergence rate for the variable stepsize Bregman operator splitting algorithm, SIAM J. Numer. Anal., 54 (2016), pp. 15351556.

[21] W. W. Hager And H. Zhang, Inexact alternating direction methods of multipliers for separable convex optimization, Comput. Optim. Appl., 73 (2019), pp. 201-235.

[22] —- Convergence rates for an inexact ADMM applied to separable convex optimization, arXiv, (2020, DOI: 2001.02503).

[23] D. Han, D. Sun, and L. Zhang, Linear rate convergence of the Alternating Direction Method of Multipliers for convex composite programming, Math. Oper. Res., 43 (2018), pp. 622637.

[24] D. Han And X. YuAn, A note on the alternating direction method of multipliers, J. Optim. Theory Appl., 155 (2012), pp. 227-238.

[25] B. He, L. LiaO, D. Han, AND H. YAn, A new inexact alternating directions method for monotone variational inequalities, Math. Programming, 92 (2002), pp. 103-118.

[26] B. He, M. TAO, M. XU, AND X. YuAN, An alternating direction-based contraction method 
for linearly constrained separable convex programming problems, Optimization, 62 (2013), pp. 573-596.

[27] B. He, M. TaO, And X. Yuan, Alternating direction method with Gaussian back substitution for separable convex programming, SIAM J. Optim., 22 (2012), pp. 313-340.

[28] B. He And X. YuAn, On the $\mathcal{O}(1 / n)$ convergence rate of the Douglas-Rachford alternating direction method, SIAM J. Numer. Anal., 50 (2012), pp. 700-709.

[29] B. S. He, F. MA, AND X. M. Yuan, Optimally linearizing the alternating direction method of multipliers for convex programming, Comput. Optim. Appl., 75 (2020), pp. 361-388.

[30] M. Hong AND Z. LuO, On the linear convergence of the alternating direction method of multipliers, Math. Program., 162 (2017), pp. 165-199.

[31] M. LI, L. LIAO, AND X. YUAN, Inexact alternating direction methods of multipliers with logarithmic-quadratic proximal regularization, J. Optim. Theory Appl., 159 (2013), pp. $412-436$.

[32] M. LI, D. Sun, And K. C. ToH, A convergent 3-block semi-proximal ADMM for for convex minimization problems with one strongly convex block, Asia-Pacific Journal of Operational Research, 32 (2015), pp. 1-19.

[33] T. Lin, S. MA, AND S. ZHANG, On the global linear convergence of the ADMM with multiblock variables, SIAM J. Optim., 25 (2015), pp. 1478-1497.

[34] Y. Liu, X. Yuan, S. Zeng, and J. Zhang, Partial error bound conditions for the linear convergence rate of the alternating direction method of multipliers, SIAM J. Numer. Anal., 56 (2018), pp. 2095-2123.

[35] R. D. C. Monteiro AND B. F. Svaiter, Iteration-complexity of block-decomposition algorithms and the alternating direction method of multipliers, SIAM J. Optim., 23 (2013), pp. 475507.

[36] S. M. Robinson, Some continuity properties of polyhedral multifunctions, Math. Prog. Study, 14 (1981), pp. 206-214.

[37] R. T. RockafELlar, Monotone operators and the proximal point algorithm, SIAM J. Control, 14 (1976), pp. 877-898.

[38] R. Shefi And M. Teboulle, Rate of convergence analysis of decomposition methods based on the proximal method of multipliers for convex minimization, SIAM J. Optim., 24 (2014), pp. 269-297.

[39] M. V. Solodov And B. F. Svaiter, An inexact hybrid generalized proximal point algorithm and some new results on the theory of Bregman functions, Math. Oper. Res., 25 (2000), pp. 214-230.

[40] M. TAO AND X. YUAN, Recovering low-rank and sparse components of matrices from incomplete and noisy observations, SIAM J. Optim., 21 (2011), pp. 57-81.

[41] Z. Wen, D. GoldFarb, AND W. Yin, Alternating direction augmented Lagrangian methods for semidefinite programming, Math. Prog. Comput., 2 (2010), pp. 203-230.

[42] W. H. YANG AND D. HAN, Linear convergence of the alternating direction method of multipliers for a class of convex optimization problems, SIAM J. Numer. Anal., 54 (2016), pp. 625-640.

[43] X. YuAn, S. Z. ZenG, AND J. ZHANG, Discerning the linear convergence of admm for structured convex optimization through the lens of variational analysis, J. Mach. Learn. Res., to appear (2020). 